\title{
Tutorial on seismic interferometry: Part 1 - Basic principles and applications
}

\author{
Kees Wapenaar ${ }^{1}$, Deyan Draganov ${ }^{1}$, Roel Snieder ${ }^{2}$, Xander Campman $^{3}$, and Arie Verdel $^{3}$
}

\begin{abstract}
Seismic interferometry involves the crosscorrelation of responses at different receivers to obtain the Green's function between these receivers. For the simple situation of an impulsive plane wave propagating along the $x$-axis, the crosscorrelation of the responses at two receivers along the $x$-axis gives the Green's function of the direct wave between these receivers. When the source function of the plane wave is a transient (as in exploration seismology) or a noise signal (as in passive seismology), then the crosscorrelation gives the Green's function, convolved with the autocorrelation of the source function. Direct-wave interferometry also holds for 2D and 3D situations, assuming the receivers are surrounded by a uniform distribution of sources. In this case, the main contributions to the retrieved direct wave between the receivers come from sources in Fresnel zones around stationary points. The main application of direct-wave interferometry is the
\end{abstract}

retrieval of seismic surface-wave responses from ambient noise and the subsequent tomographic determination of the surfacewave velocity distribution of the subsurface. Seismic interferometry is not restricted to retrieving direct waves between receivers. In a classic paper, Claerbout shows that the autocorrelation of the transmission response of a layered medium gives the plane-wave reflection response of that medium. This is essentially $1 \mathrm{D}$ reflected-wave interferometry. Similarly, the crosscorrelation of the transmission responses, observed at two receivers, of an arbitrary inhomogeneous medium gives the $3 \mathrm{D}$ reflection response of that medium. One of the main applications of reflected-wave interferometry is retrieving the seismic reflection response from ambient noise and imaging of the reflectors in the subsurface. A common aspect of direct- and reflected-wave interferometry is that virtual sources are created at positions where there are only receivers without requiring knowledge of the subsurface medium parameters or of the positions of the actual sources.

\section{INTRODUCTION}

In this two-part tutorial, we give an overview of the basic principles and the underlying theory of seismic interferometry and discuss applications and new advances. The term seismic interferometry refers to the principle of generating new seismic responses of virtual sources ${ }^{4}$ by crosscorrelating seismic observations at different receiver locations. One can distinguish between controlled-source and passive seismic interferometry. Controlled-source seismic interferometry, pioneered by Schuster (2001), Bakulin and Calvert (2004), and others, comprises a new processing methodology for seismic exploration data. Apart from crosscorrelation, controlled-source interferometry also involves summation of correlations over different source positions. Passive seismic interferometry, on the other hand, is a methodology for turning passive seismic measurements (ambient seismic noise or microearthquake responses) into deterministic seismic responses. Here, we further distinguish between retrieving surface-wave transmission responses (Campillo and Paul, 2003; Shapiro and Campillo, 2004; Sabra, Gerstoft, et al., 2005a) and exploration reflection responses (Claerbout, 1968; Scherbaum, 1987b; Draganov et al., 2007, 2009). In passive interferometry of ambient noise, no explicit summation of correlations over different source positions is required because the correlated responses are a superposition of simultaneously acting uncorrelated sources.

In all cases, the response that is retrieved by crosscorrelating two

Manuscript received by the Editor 30 November 2009; published online 14 September 2010.

${ }^{1}$ Delft University of Technology, Department of Geotechnology, Delft, The Netherlands.E-mail: c.p.a.wapenaar@tudelft.nl; d.s.draganov@tudelft.nl.

${ }^{2}$ Colorado School of Mines, Center for Wave Phenomena, Golden, Colorado, U.S.A. E-mail: rsnieder@ mines.edu.

${ }^{3}$ Shell International Exploration and Production, Rijswijk, The Netherlands. E-mail: xander.campman@ @ shell.com; arie.verdel@gmail.com. (C) 2010 Society of Exploration Geophysicists. All rights reserved.

${ }^{4}$ In the literature on seismic interferometry, the term virtual source often refers to the method of Bakulin and Calvert $(2004,2006)$, which is discussed extensively in Part 2. However, creating a virtual source is the essence of nearly all seismic interferometry methods (see e.g., Schuster (2001), who already used this term) In this paper (Parts 1 and 2) we use the term virtual source whenever appropriate. When it refers to Bakulin and Calvert's method, we will mention this explicitly. 
receiver recordings (and summing over different sources) can be interpreted as the response that would be measured at one of the receiver locations as if there were a source at the other. Because such a point-source response is equal to a Green's function convolved with a wavelet, seismic interferometry is also often called Green's function retrieval. Both terms are used in this paper. The term interferometry is borrowed from radio astronomy, where it refers to crosscorrelation methods applied to radio signals from distant objects (Thompson et al., 2001). The name Green's function honors George Green who, in a privately published essay, introduced the use of impulse responses in field representations (Green, 1828). Challis and Sheard (2003) give a brief history of Green's life and theorem. Ramírez and Weglein (2009) review applications of Green's theorem in seismic processing.

Early successful results of Green's function retrieval from noise correlations were obtained in the field of ultrasonics (Weaver and Lobkis, 2001, 2002). The experiments were done with diffuse fields in a closed system. Here diffuse means that the amplitudes of the normal modes are uncorrelated but have equal expected energies. Hence, the crosscorrelation of the field at two receiver positions does not contain cross-terms of unequal normal modes. The sum of the remaining terms is proportional to the modal representation of the Green's function of the closed system (Lobkis and Weaver, 2001). This means that the crosscorrelation of a diffuse field in a closed system converges to its impulse response. Later, it was recognized (e.g., Godin, 2007) that this theoretical explanation is akin to the fluctuation-dissipation theorem (Callen and Welton, 1951; Rytov, 1956; Rytov et al., 1989; Le Bellac et al., 2004).

The earth is a closed system; but at the scale of global seismology, the wavefield is far from diffuse. At the scale of exploration seismology, an ambient-noise field may have a diffuse character, but the encompassing system is not closed. Hence, for seismic interferometry, the normal-mode approach breaks down. Throughout this paper, we consider seismic interferometry (or Green's function retrieval) in open systems, including half-spaces below a free surface. Instead of a treatment per field of application or a chronological discussion, we have chosen a setup in which we explain the principles of seismic interferometry step by step. In Part 1, we start with the basic principles

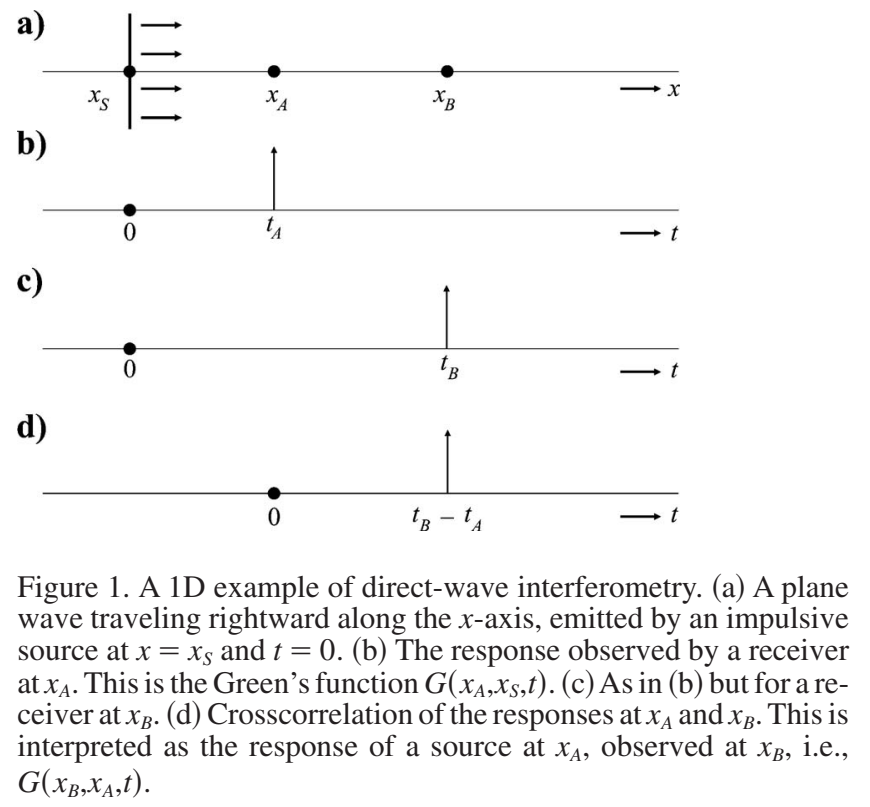

of 1D direct-wave interferometry and conclude with a discussion of the principles of 3D reflected-wave interferometry. We present applications in controlled-source as well as passive interferometry and, where appropriate, review the historical background. To stay focused on seismic applications, we refrain from a further discussion of the normal-mode approach, nor do we address the many interesting applications of Green's function retrieval in underwater acoustics (e.g., Roux and Fink, 2003; Sabra et al., 2005; Brooks and Gerstoft, 2007).

\section{DIRECT-WAVE INTERFEROMETRY}

\section{D analysis of direct-wave interferometry}

We start our explanation of seismic interferometry by considering an illustrative 1D analysis of direct-wave interferometry. Figure 1a shows a plane wave, radiated by an impulsive unit source at $x=x_{S}$ and $t=0$, propagating in the rightward direction along the $x$-axis. We assume that the propagation velocity $c$ is constant and the medium is lossless. There are two receivers along the $x$-axis at $x_{A}$ and $x_{B}$. Figure $1 \mathrm{~b}$ shows the response observed by the first receiver at $x_{A}$. We denote this response as $G\left(x_{A}, x_{S}, t\right)$, where $G$ stands for the Green's function. Throughout this paper, we use the common convention that the first two arguments in $G\left(x_{A}, x_{S}, t\right)$ denote the receiver and source coordinates, respectively (here, $x_{A}$ and $x_{S}$ ), whereas the last argument denotes time $t$ or angular frequency $\omega$. In our example, this Green's function consists of an impulse at $t_{A}=\left(x_{A}-x_{S}\right) / c$; therefore, $G\left(x_{A}, x_{S}, t\right)=\delta\left(t-t_{A}\right)$, where $\delta(t)$ is the Dirac delta function. Similarly, the response at $x_{B}$ is given by $G\left(x_{B}, x_{S}, t\right)=\delta\left(t-t_{B}\right)$, with $t_{B}=\left(x_{B}-x_{S}\right) / c$ (Figure 1c).

Seismic interferometry involves the crosscorrelation of responses at two receivers, in this case at $x_{A}$ and $x_{B}$. Looking at Figure 1a, it appears that the raypaths associated with $G\left(x_{A}, x_{S}, t\right)$ and $G\left(x_{B}, x_{S}, t\right)$ have the path from $x_{S}$ to $x_{A}$ in common. The traveltime along this common path cancels in the crosscorrelation process, leaving the traveltime along the remaining path from $x_{A}$ to $x_{B}$, i.e., $t_{B}-t_{A}=\left(x_{B}\right.$ $\left.-x_{A}\right) / c$. Hence, the crosscorrelation of the responses in Figure $1 \mathrm{~b}$ and $\mathrm{c}$ is an impulse at $t_{B}-t_{A}$ (see Figure 1d). This impulse can be interpreted as the response of a source at $x_{A}$ observed by a receiver at $x_{B}$, i.e., the Green's function $G\left(x_{B}, x_{A}, t\right)$. An interesting observation is that the propagation velocity $c$ and the position of the actual source $x_{S}$ need not be known. The traveltimes along the common path from $x_{S}$ to $x_{A}$ compensate each other, independent of the propagation velocity and the length of this path. Similarly, if the source impulse would occur at $t=t_{S}$ instead of at $t=0$, the impulses observed at $x_{A}$ and $x_{B}$ would be shifted by the same amount of time $t_{S}$, which would be canceled in the crosscorrelation. Thus, the absolute time $t_{S}$ at which the source emits its pulse need not be known.

Let us discuss this example a bit more precisely. We denote the crosscorrelation of the impulse responses at $x_{A}$ and $x_{B}$ as $G\left(x_{B}, x_{S}, t\right) * G\left(x_{A}, x_{S},-t\right)$. The asterisk denotes temporal convolution, but the time reversal of the second Green's function turns the convolution into a correlation, defined as $G\left(x_{B}, x_{S}, t\right) * G\left(x_{A}, x_{S},-t\right)$ $=\int G\left(x_{B}, x_{S}, t+t^{\prime}\right) G\left(x_{A}, x_{S}, t^{\prime}\right) \mathrm{d} t^{\prime}$. Substituting the delta functions into the right-hand side gives $\int \delta\left(t+t^{\prime}-t_{B}\right) \delta\left(t^{\prime}-t_{A}\right) \mathrm{d} t^{\prime}=\delta(t$ $\left.-\left(t_{B}-t_{A}\right)\right)=\delta\left(t-\left(x_{B}-x_{A}\right) / c\right)$. This is indeed the Green's function $G\left(x_{B}, x_{A}, t\right)$, propagating from $x_{A}$ to $x_{B}$. Because we started this derivation with the crosscorrelation of the Green's functions, we have obtained the following 1D Green's function representation: 


$$
G\left(x_{B}, x_{A}, t\right)=G\left(x_{B}, x_{S}, t\right) * G\left(x_{A}, x_{S},-t\right) .
$$

This representation formulates the principle that the crosscorrelation of observations at two receivers $\left(x_{A}\right.$ and $\left.x_{B}\right)$ gives the response at one of those receivers $\left(x_{B}\right)$ as if there were a source at the other receiver $\left(x_{A}\right)$. It also shows why seismic interferometry is often called Green's function retrieval.

Note that the source is not necessarily an impulse. If the source function is defined by some wavelet $s(t)$, then the responses at $x_{A}$ and $x_{B}$ can be written as $u\left(x_{A}, x_{S}, t\right)=G\left(x_{A}, x_{S}, t\right) * s(t)$ and $u\left(x_{B}, x_{S}, t\right)$ $=G\left(x_{B}, x_{S}, t\right) * s(t)$, respectively. Let $S_{s}(t)$ be the autocorrelation of the wavelet, i.e., $S_{s}(t)=s(t) * s(-t)$. Then the crosscorrelation of $u\left(x_{A}, x_{S}, t\right)$ and $u\left(x_{B}, x_{S}, t\right)$ gives the right-hand side of equation 1 , convolved with $S_{s}(t)$. This is equal to the left-hand side of equation 1 , convolved with $S_{s}(t)$. Therefore,

$$
G\left(x_{B}, x_{A}, t\right) * S_{s}(t)=u\left(x_{B}, x_{S}, t\right) * u\left(x_{A}, x_{S},-t\right) .
$$

In words: If the source function is a wavelet instead of an impulse, then the crosscorrelation of the responses at two receivers gives the Green's function between these receivers, convolved with the autocorrelation of the source function.

This principle holds true for any source function, including noise. Figure $2 \mathrm{a}$ and $\mathrm{b}$ shows the responses at $x_{A}$ and $x_{B}$, respectively, of a bandlimited noise source $N(t)$ at $x_{S}$ (the central frequency of the noise is $30 \mathrm{~Hz}$; the figure shows only $4 \mathrm{~s}$ of a total of $160 \mathrm{~s}$ of noise). In this numerical example, the distance between the receivers is $1200 \mathrm{~m}$ and the propagation velocity is $2000 \mathrm{~m} / \mathrm{s}$; hence, the traveltime between these receivers is $0.6 \mathrm{~s}$. As a consequence, the noise response at $x_{B}$ in Figure $2 \mathrm{~b}$ is $0.6 \mathrm{~s}$ delayed with respect to the response at $x_{A}$ in Figure $2 \mathrm{a}$ (similar to the impulse in Figure 1c delayed with respect to the impulse in Figure 1b). Crosscorrelation of these noise responses gives, analogous to equation 2 , the impulse response be-

a)

b)
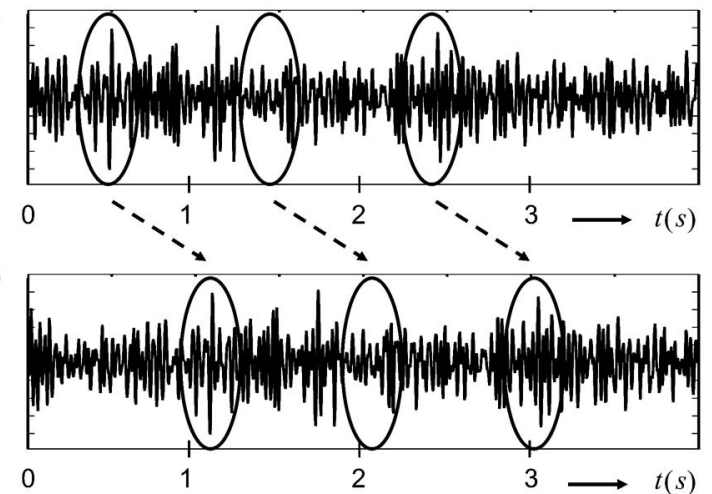

c)

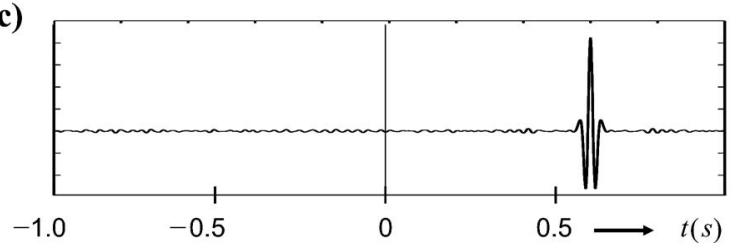

Figure 2. As in Figure 1 but this time for a noise source $N(t)$ at $x_{S}$. (a) The response observed at $x_{A}$, i.e., $u\left(x_{A}, x_{S}, t\right)=G\left(x_{A}, x_{S}, t\right) * N(t)$. (b) As in (a) but for a receiver at $x_{B}$. (c) The crosscorrelation, which is equal to $G\left(x_{B}, x_{A}, t\right) * S_{N}(t)$, with $S_{N}(t)$ the autocorrelation of the noise. tween $x_{A}$ and $x_{B}$, convolved with $S_{N}(t)$, i.e., the autocorrelation of the noise $N(t)$. The correlation is shown in Figure 2c, which indeed reveals a bandlimited impulse centered at $t=0.6 \mathrm{~s}$ (the traveltime from $x_{A}$ to $x_{B}$ ). Note that from registrations at two receivers of a noise field from an unknown source in a medium with unknown propagation velocity, we have obtained a bandlimited version of the Green's function. By dividing the distance between the receivers $(1200 \mathrm{~m})$ by the traveltime estimated from the bandlimited Green's function $(0.6 \mathrm{~s})$, we obtain an estimate of the propagation velocity between the receivers $(2000 \mathrm{~m} / \mathrm{s})$. This illustrates that direct-wave interferometry can be used for tomographic inversion.

Until now, we considered a single plane wave propagating in the positive $x$-direction. In Figure $3 \mathrm{a}$, we consider the same configuration as in Figure 1a, but now an impulsive unit source at $x=x_{S}^{\prime}$ radiates a leftward-propagating plane wave. Figure $3 \mathrm{~b}$ is the response at $x_{A}$, given by $G\left(x_{A}, x_{S}^{\prime}, t\right)=\delta\left(t-t_{A}^{\prime}\right)$, with $t_{A}^{\prime}=\left(x_{S}^{\prime}-x_{A}\right) / c$. Similarly, the response at $x_{B}$ is $G\left(x_{B}, x_{S}^{\prime}, t\right)=\delta\left(t-t_{B}^{\prime}\right)$, with $t_{B}^{\prime}=\left(x_{S}^{\prime}-x_{B}\right) / c$ (Figure 3c). The crosscorrelation of these responses gives $\delta\left(t-\left(t_{B}^{\prime}\right.\right.$ $\left.\left.-t_{A}^{\prime}\right)\right)=\delta\left(t+\left(x_{B}-x_{A}\right) / c\right)$, which is equal to the time-reversed Green's function $G\left(x_{B}, x_{A},-t\right)$. So, for the configuration of Figure $3 \mathrm{a}$, we obtain the following Green's function representation:

$$
G\left(x_{B}, x_{A},-t\right)=G\left(x_{B}, x_{S}^{\prime}, t\right) * G\left(x_{A}, x_{S}^{\prime},-t\right) .
$$

We can combine equations 1 and 3 as follows:

$$
G\left(x_{B}, x_{A}, t\right)+G\left(x_{B}, x_{A},-t\right)=\sum_{i=1}^{2} G\left(x_{B}, x_{S}^{(i)}, t\right) * G\left(x_{A}, x_{S}^{(i)},-t\right),
$$

where $x_{S}^{(i)}$ for $i=1,2$ stands for $x_{S}$ and $x_{S}^{\prime}$, respectively.

For the 1D situation, this combination may not seem very useful. We analyze it here, however, because this representation better resembles the $2 \mathrm{D}$ and $3 \mathrm{D}$ representations we encounter later. Note that because $G\left(x_{B}, x_{A}, t\right)$ is the causal response of an impulse at $t=0$ (meaning it is nonzero only for $t>0$ ), it does not overlap with $G\left(x_{B}, x_{A},-t\right)$ (which is nonzero only for $t<0$ ). Hence, $G\left(x_{B}, x_{A}, t\right)$ can be resolved from the left-hand side of equation 4 by extracting the causal part. If the source function is a wavelet $s(t)$ with autocorrelation $S_{s}(t)$, we obtain, analogous to equation 2 ,

a)

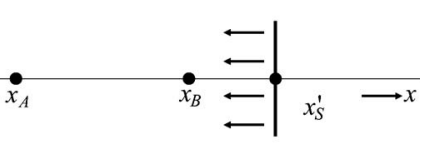

b)

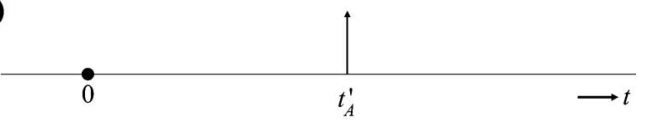

c)

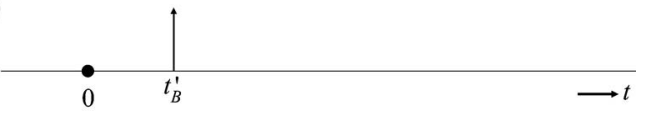

d)

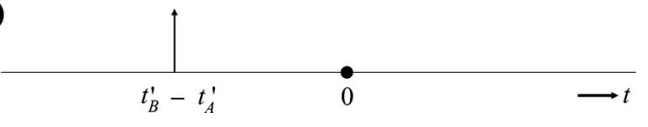

Figure 3. As in Figure 1 but this time for a leftward-traveling impulsive plane wave. The crosscorrelation in (d) is interpreted as the time-reversed Green's function $G\left(x_{B}, x_{A},-t\right)$. 


$$
\begin{array}{r}
\left\{G\left(x_{B}, x_{A}, t\right)+G\left(x_{B}, x_{A},-t\right)\right\} * S_{S}(t) \\
\quad=\sum_{i=1}^{2} u\left(x_{B}, x_{S}^{(i)}, t\right) * u\left(x_{A}, x_{S}^{(i)},-t\right) .
\end{array}
$$

Here, $G\left(x_{B}, x_{A}, t\right) * S_{s}(t)$ may have some overlap with $G\left(x_{B}, x_{A}\right.$, $-t) * S_{s}(t)$ for small $|t|$, depending on the length of the autocorrelation function $S_{s}(t)$. Therefore, $G\left(x_{B}, x_{A}, t\right) * S_{s}(t)$ can be extracted from the left-hand side of equation 5, except for small distances $\mid x_{B}$ $-x_{A} \mid$.

The right-hand sides of equations 4 and 5 state that the crosscorrelation is applied to the responses of each source separately, after which the summation over the sources is carried out. For impulsive sources or transient wavelets $s(t)$, these steps should not be interchanged. Let us see why. Suppose the sources at $x_{S}$ and $x_{S}^{\prime}$ act simultaneously, as illustrated in Figure $4 \mathrm{a}$. Then the response at $x_{A}$ would be given by $u\left(x_{A}, t\right)=\Sigma_{i=1}^{2} G\left(x_{A}, x_{S}^{(i)}, t\right) * s(t)$ and the response at $x_{B}$ by $u\left(x_{B}, t\right)=\sum_{j=1}^{2} G\left(x_{B}, x_{S}^{(j)}, t\right) * s(t)$. These responses are shown in Figure $4 \mathrm{~b}$ and $\mathrm{c}$ for an impulsive source $(s(t)=\delta(t))$. The crosscorrelation of these responses, shown in Figure $4 \mathrm{~d}$, contains two crossterms at $t_{B}-t_{A}^{\prime}$ and $t_{B}^{\prime}-t_{A}$ that have no physical meaning. Hence, for impulsive or transient sources, the order of crosscorrelation and summation matters.

The situation is different for noise sources. Consider two simultaneously acting noise sources $N_{1}(t)$ and $N_{2}(t)$ at $x_{S}$ and $x_{S}^{\prime}$, respectively. The responses at $x_{A}$ and $x_{B}$ are given by $u\left(x_{A}, t\right)$ $=\sum_{i=1}^{2} G\left(x_{A}, x_{S}^{(i)}, t\right) * N_{i}(t)$ and $u\left(x_{B}, t\right)=\sum_{j=1}^{2} G\left(x_{B}, x_{S}^{(j)}, t\right) * N_{j}(t)$, respectively (see Figure $5 \mathrm{a}$ and $\mathrm{b}$ ). Because each of these responses is the superposition of a rightward- and a leftward-propagating wave, the response in Figure 5b is not a shifted version of that in Figure 5a (unlike the responses in Figure $2 \mathrm{a}$ and $\mathrm{b}$ ). We assume that the noise sources are uncorrelated; thus, $\left\langle N_{j}(t) * N_{i}(-t)\right\rangle=\delta_{i j} S_{N}(t)$, where $\delta_{i j}$ is the Kronecker delta function and $\langle\cdot\rangle$ denotes ensemble averaging. In practice, the ensemble averaging is replaced by integrating over sufficiently long time. In the numerical example the duration of the noise signals is again $160 \mathrm{~s}$ (only $4 \mathrm{~s}$ of noise is shown in Figure $5 \mathrm{a}$ and $\mathrm{b}$ ). For the crosscorrelation of the responses at $x_{A}$ and $x_{B}$, we can now write

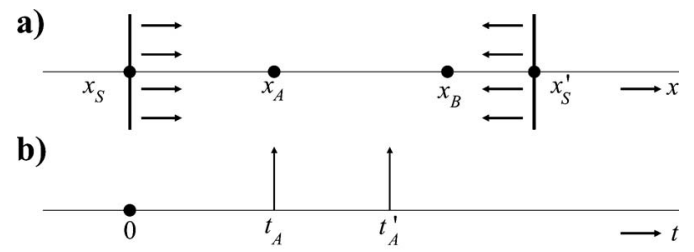

c)

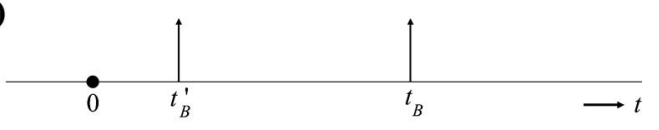

d)

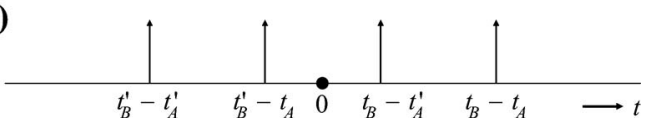

Figure 4. As in Figures 1 and 3 but with simultaneously rightwardand leftward-traveling impulsive plane waves. The crosscorrelation in (d) contains cross-terms that have no physical meaning.

$$
\begin{aligned}
\left\langle u\left(x_{B}, t\right)\right. & \left.* u\left(x_{A},-t\right)\right\rangle \\
= & \left\langle\sum_{j=1}^{2} \sum_{i=1}^{2} G\left(x_{B}, x_{S}^{(j)}, t\right) * N_{j}(t)\right. \\
& \left.* G\left(x_{A}, x_{S}^{(i)},-t\right) * N_{i}(-t)\right\rangle \\
= & \sum_{i=1}^{2} G\left(x_{B}, x_{S}^{(i)}, t\right) * G\left(x_{A}, x_{S}^{(i)},-t\right) * S_{N}(t) .
\end{aligned}
$$

Combining equation 6 with equation 4, we finally obtain

$$
\left\{G\left(x_{B}, x_{A}, t\right)+G\left(x_{B}, x_{A},-t\right)\right\} * S_{N}(t)=\left\langle u\left(x_{B}, t\right) * u\left(x_{A},-t\right)\right\rangle .
$$

Expression 7 shows that the crosscorrelation of two observed fields at $x_{A}$ and $x_{B}$, each of which is the superposition of rightward- and leftward-propagating noise fields, gives the Green's function between $x_{A}$ and $x_{B}$ plus its time-reversed version, convolved with the autocorrelation of the noise (see Figure $5 \mathrm{c}$ ). The cross-terms, unlike Figure $4 \mathrm{~d}$, do not contribute because the noise sources $N_{1}(t)$ and $N_{2}(t)$ are uncorrelated.

Miyazawa et al. (2008) apply equation 7 with $x_{A}$ and $x_{B}$ at different depths along a borehole in the presence of industrial noise at Cold Lake, Alberta, Canada. By choosing for $u$ different components of multicomponent sensors in the borehole, they retrieve separate Green's functions for P- and S-waves, the latter with different polarizations. From the arrival times in the Green's functions, they derive the different propagation velocities and accurately quantify shearwave splitting.

Despite the relative simplicity of our 1D analysis of direct-wave interferometry, we can make several observations about seismic interferometry that also hold true for more general situations. First, we can distinguish between interferometry for impulsive or transient

a)

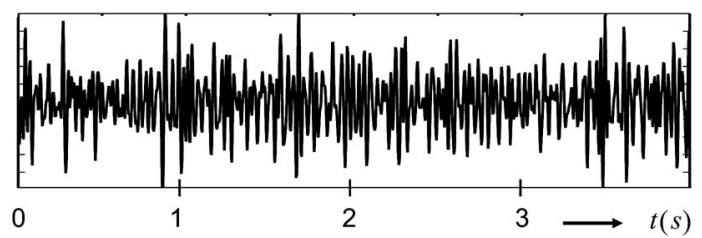

b)

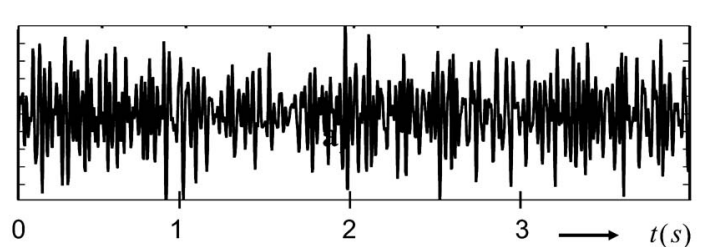

c)

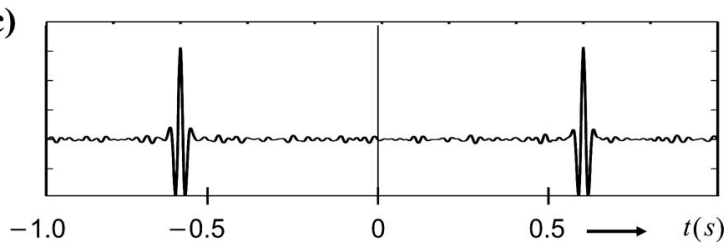

Figure 5. As in Figure 4 but this time with simultaneously rightwardand leftward-traveling uncorrelated noise fields. The crosscorrelation in (c) contains no cross-terms. 
sources on the one hand (equations 4 and 5) and interferometry for noise sources on the other hand (equation 7). In the case of impulsive or transient sources, the responses of each source must be crosscorrelated separately, after which a summation over the sources takes place. In the case of uncorrelated noise sources, a single crosscorrelation suffices.

Second, it appears that an isotropic illumination of the receivers is required to obtain a time-symmetric response between the receivers (of which the causal part is the actual response). In one dimension, isotropic illumination means equal illumination by rightward- and leftward-propagating waves. In two and three dimensions, it means equal illumination from all directions (discussed in the next section).

Finally, instead of the time-symmetric response $G\left(x_{B}, x_{A}, t\right)+G\left(x_{B}, x_{A},-t\right)$, in the literature we often encounter an antisymmetric response $G\left(x_{B}, x_{A}, t\right)-G\left(x_{B}, x_{A},-t\right)$. This is merely a result of differently defined Green's functions. Note that a simple time differentiation of the Green's functions would turn the symmetric response into an antisymmetric one, and vice versa (see Wapenaar and Fokkema [2006] for a more detailed discussion on this aspect)

\section{D and 3D analysis of direct-wave interferometry}

We extend our discussion of direct-wave interferometry to configurations with more dimensions. In the following discussion, we mainly use heuristic arguments, illustrated with a numerical example. For a more precise derivation based on stationary-phase analysis, we refer to Snieder (2004).

Consider the 2D configuration shown in Figure 6a. The horizontal dashed line corresponds to the 1D configuration of Figure 1a, with two receivers at $\mathbf{x}_{A}$ and $\mathbf{x}_{B}, 1200 \mathrm{~m}$ apart ( $\mathbf{x}$ denotes a Cartesian coordinate vector). The propagation velocity $c$ is $2000 \mathrm{~m} / \mathrm{s}$, and the medium is again assumed to be lossless. Instead of plane-wave sources, we have many point sources denoted by the small black dots, distributed over a "pineapple slice," emitting transient signals with a central frequency of $30 \mathrm{~Hz}$. In polar coordinates, the positions of the sources are denoted by $\left(r_{S}, \phi_{S}\right)$. The angle $\phi_{S}$ is equidistantly sampled $\left(\Delta \phi_{S}=0.25^{\circ}\right)$, whereas the distance $r_{S}$ to the center of the slice is chosen randomly between 2000 and $3000 \mathrm{~m}$. The responses at the two receivers at $\mathbf{x}_{A}$ and $\mathbf{x}_{B}$ are shown in Figure $6 \mathrm{~b}$ and c, respectively, as a function of the (polar) source coordinate $\phi_{S}$ (for display purposes, only every sixteenth trace is shown). These responses are crosscorrelated (for each source separately), and the crosscorrelations are shown in Figure 6d, again as a function of $\phi_{S}$. Such a gather is often called a correlation gather. Note that the traveltimes in this correlation gather vary smoothly with $\phi_{S}$, despite the randomness of the traveltimes in Figure $6 \mathrm{~b}$ and $\mathrm{c}$. This is because in the crosscorrelation process only the time difference along the paths to $\mathbf{x}_{A}$ and $\mathbf{x}_{B}$ matters.

The source in Figure $6 \mathrm{a}$ with $\phi_{S}=0^{\circ}$ plays the same role as the plane-wave source at $x_{S}$ in Figure 1a. For this source, the crosscorrelation gives a signal at $\left|\mathbf{x}_{B}-\mathbf{x}_{A}\right| / c=0.6 \mathrm{~s}$, seen in the trace at $\phi_{S}$ $=0^{\circ}$ in Figure $6 \mathrm{~d}$. Similarly, the source at $\phi_{S}=180^{\circ}$ plays the same role as the plane-wave source at $x_{S}^{\prime}$ in Figure $3 \mathrm{a}$ and leads to the trace at $\phi_{S}=180^{\circ}$ in Figure $6 \mathrm{~d}$ with a signal at $-0.6 \mathrm{~s}$. Analogous to equation 5 , we sum the crosscorrelations of all sources, i.e., we sum
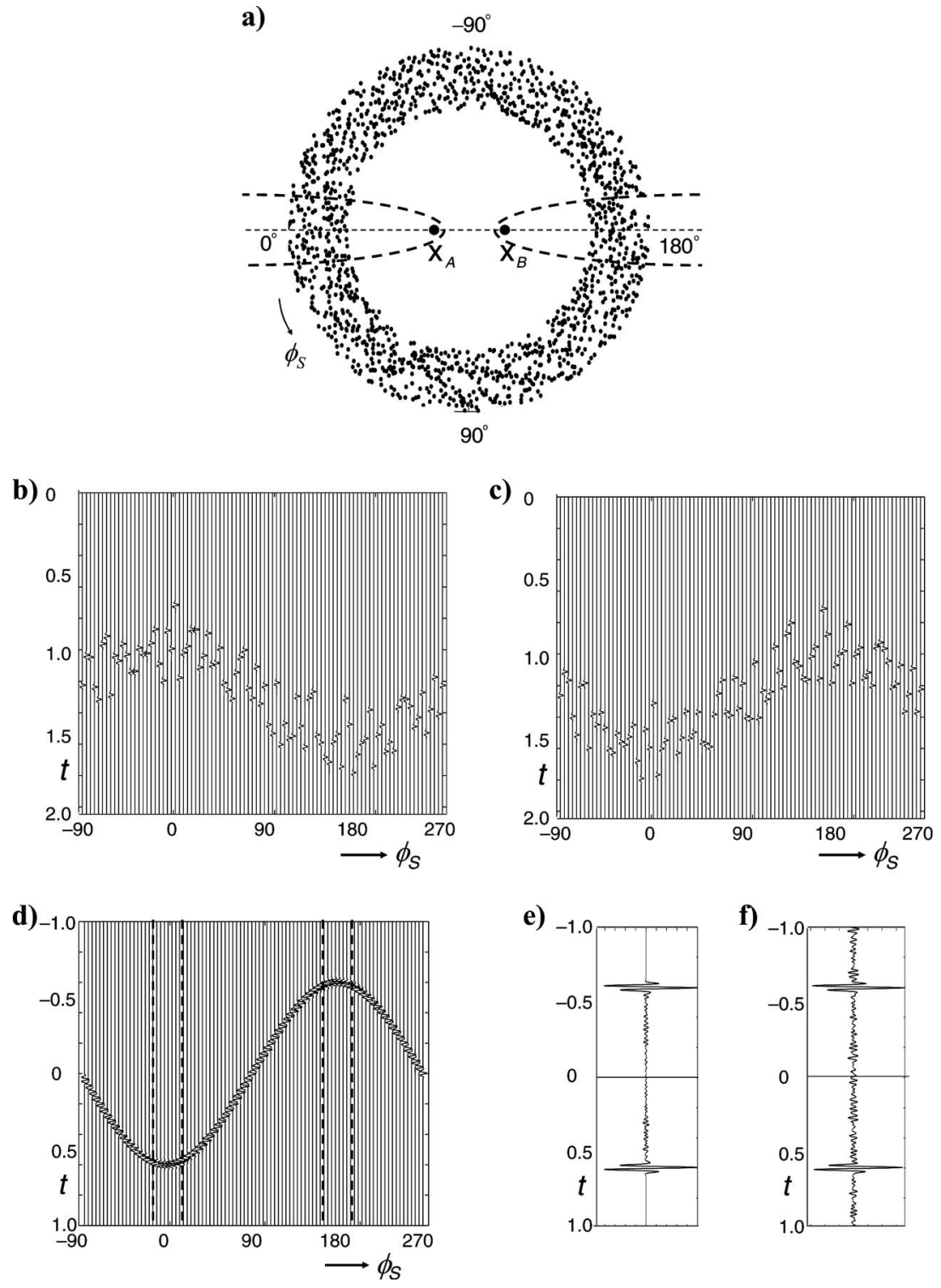

Figure 6. A 2D example of direct-wave interferometry. (a) Distribution of point sources, isotropically illuminating the receivers at $\mathbf{x}_{A}$ and $\mathbf{x}_{B}$. The thick dashed lines indicate the Fresnel zones. (b) Responses at $\mathbf{x}_{A}$ as a function of the (polar) source coordinate $\phi_{S}$. (c) Responses at $\mathbf{x}_{B}$. (d) Crosscorrelation of the responses at $\mathbf{x}_{A}$ and $\mathbf{x}_{B}$. The dashed lines indicate the Fresnel zones. (e) The sum of the correlations in (d). This is interpreted as $\left\{G\left(\mathbf{x}_{B}, \mathbf{x}_{A}, t\right)+G\left(\mathbf{x}_{B}, \mathbf{x}_{A},-t\right)\right\} * S_{s}(t)$. The main contributions come from sources in the Fresnel zones indicated in (a) and (d). (f) Single crosscorrelation of the responses at $\mathbf{x}_{A}$ and $\mathbf{x}_{B}$ of simultaneously acting uncorrelated noise sources. The duration of the noise signals was $9600 \mathrm{~s}$. 
all traces in Figure 6d, which leads to the time-symmetric response in Figure $6 \mathrm{e}$, with two events at 0.6 and $-0.6 \mathrm{~s}$. These two events are again interpreted as the response of a source at $\mathbf{x}_{A}$, observed at $\mathbf{x}_{B}$, plus its time-reversed version, i.e., $\left\{G\left(\mathbf{x}_{B}, \mathbf{x}_{A}, t\right)+G\left(\mathbf{x}_{B}, \mathbf{x}_{A}\right.\right.$, $-t)\} * S_{s}(t)$, where $S_{s}(t)$ is the autocorrelation of the source wavelet. Because the sources have a finite frequency content, not only do the sources exactly at $\phi_{S}=0^{\circ}$ and $\phi_{S}=180^{\circ}$ contribute to these events but also the sources in Fresnel zones around these angles. These Fresnel zones are denoted by the thick dashed lines in Figure 6a and d. In Figure 6d, the centers of these Fresnel zones are the stationary points of the traveltime curve of the crosscorrelations. Note that the events in all traces outside the Fresnel zones in Figure 6d interfere destructively and give no coherent contribution in Figure 6e. The noise between the two events in Figure 6e results because the traveltime curve in Figure $6 \mathrm{~d}$ is not $100 \%$ smooth, caused by the randomness of the source positions in Figure 6a.

The response in Figure 6e is obtained by summing crosscorrelations of independent transient sources. Using the arguments in the previous section, we can replace the transient sources with simultaneously acting noise sources. The cross-terms disappear when the noise sources are uncorrelated; hence, a single crosscorrelation of noise observations at $\mathbf{x}_{A}$ and $\mathbf{x}_{B}$ gives, analogous to equation 7 , $\left\{G\left(\mathbf{x}_{B}, \mathbf{x}_{A}, t\right)+G\left(\mathbf{x}_{B}, \mathbf{x}_{A},-t\right)\right\} * S_{N}(t)$, where $S_{N}(t)$ is the autocorrelation of the noise (see Figure $6 \mathrm{f}$ ). Note that the symmetry of the responses in Figure 6e and $\mathrm{f}$ relies again on the isotropic illumination of the receivers, i.e., on the net power flux of the illuminating wavefield being (close to) zero (van Tiggelen, 2003; Malcolm et al., 2004; Sánchez-Sesma et al., 2006; Snieder et al., 2007; Perton et al., 2009; Weaver et al., 2009; Yao et al., 2009).

Of course, what is demonstrated here for a $2 \mathrm{D}$ distribution of sources also holds for a $3 \mathrm{D}$ source distribution. In that case, all sources in Fresnel volumes rather than Fresnel zones contribute to the retrieval of the direct wave between $\mathbf{x}_{A}$ and $\mathbf{x}_{B}$. Furthermore, the

a)

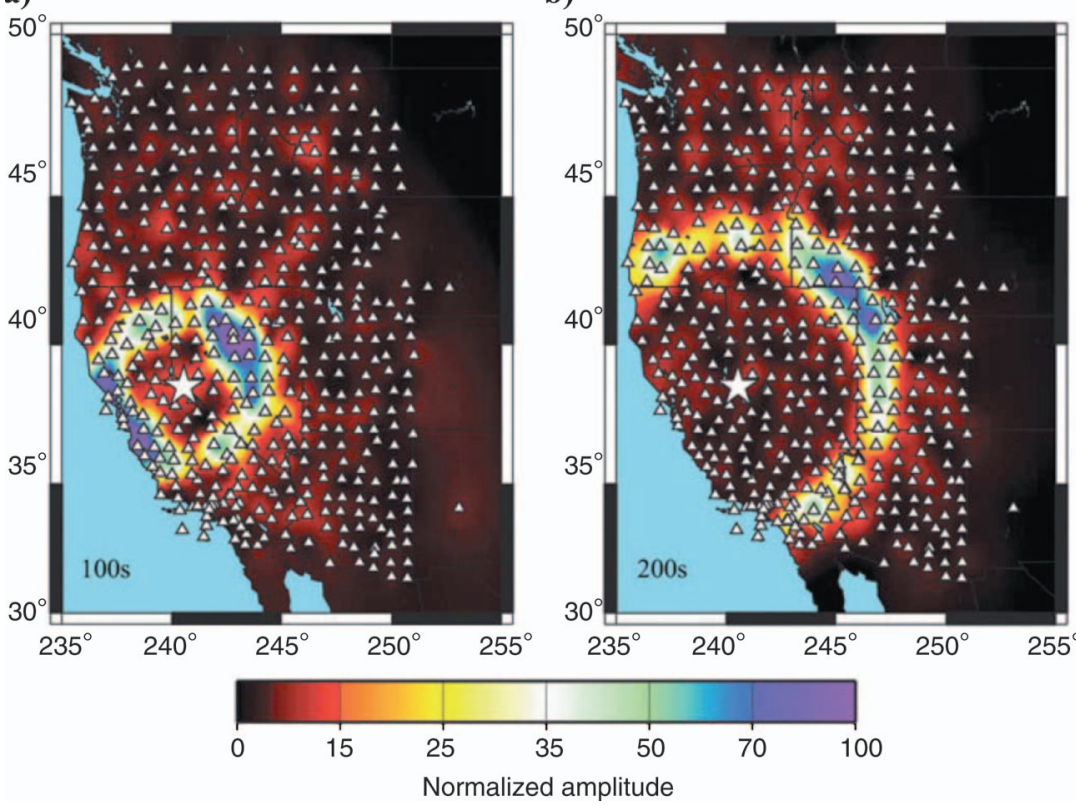

Figure 7. Two snapshots of the Rayleigh-wave response of a virtual source (the white star) southeast of Lake Tahoe, California, U.S.A. (Lin et al., 2009). The white triangles represent more than 400 seismometers (USArray stations). The shown response was obtained by crosscorrelating three years of ambient noise, recorded at the station denoted by the star, with that recorded at all other stations. sources (in two or three dimensions) are not necessarily primary sources but can also be secondary sources, i.e., scatterers in a homogeneous embedding. These secondary sources are not independent, but the late coda of the multiply scattered response reasonably resembles a diffuse wavefield. Thus, in situations with few primary sources but many secondary sources, only the late coda is used for Green's function retrieval (Campillo and Paul, 2003). It is, however, unclear how well a scattering medium should be illuminated by different sources for the scatterers to act as independent secondary sources. Fan and Snieder (2009) show an example where the scattered waves excited by a single source are equipartitioned, in the sense that energy propagates equally in all directions, but where the crosscorrelation of those scattered waves does not resemble the Green's function.

One of the most widely used applications of direct-wave interferometry is the retrieval of seismic surface waves between seismometers and the subsequent tomographic determination of the surfacewave velocity distribution of the subsurface. This approach has been pioneered by Campillo and Paul (2003); Shapiro and Campillo (2004); Sabra et al. (2005a, 2005b); and Shapiro et al. (2005). In layered media, surface waves consist of several propagating modes, of which the fundamental mode is usually the strongest. As long as only the fundamental mode is considered, surface waves can be seen as an approximate solution of a 2D wave equation with a frequency-dependent propagation velocity. So by considering the $2 \mathrm{D}$ configuration of Figure $6 \mathrm{a}$ as a plan view, the analysis above holds for ambient surface-wave noise. The Green's function of the fundamental mode of the direct surface wave can thus be extracted by crosscorrelating ambient-noise recordings at two seismometers. When many seismometers are available, this procedure can be repeated for any combination of two seismometers. In other words, each seismometer can be turned into a virtual source, the response of which is observed by all other seismometers.

Figure 7, reproduced with permission from Lin et al. (2009), shows a beautiful example of the Rayleigh-wave response of a virtual source southeast of Lake Tahoe, California, U.S.A. The white triangles represent more than 400 seismometers (USArray stations). Ocean-generated ambient seismic noise (Longuet-Higgins, 1950; Webb, 1998; Stehly et al., 2006) was recorded between October 2004 and November 2007. Because this noise is coming from the ocean, it is far from isotropic. This means the crosscorrelation of the noise between any two stations does not yield time-symmetric results such as those in Figure 6. However, as long as one of the Fresnel zones is sufficiently covered with sources, it is possible to retrieve either $G\left(\mathbf{x}_{B}, \mathbf{x}_{A}, t\right) * S_{N}(t)$ or $G\left(\mathbf{x}_{B}, \mathbf{x}_{A},-t\right) * S_{N}(t)$ (note that the location and shape of the Fresnel zone is different for each combination of stations). The snapshots shown in Figure $7 \mathrm{a}$ and $\mathrm{b}$ were obtained by crosscorrelating the noise recorded at the station denoted by the star with noise recorded at all other stations. The amplitudes exhibit azimuthal variation due to the anisotropic illumination. Responses such as this are used for tomographic inversion of the Rayleigh-wave velocity of the crust and for measuring azimuthal anisotropy in the crust. 
Bensen et al. (2007) show that it is possible to retrieve the Rayleigh-wave velocity as a function of frequency. Brenguier et al. (2007) combine these approaches to 3D tomographic inversion. From noise measurements at the Piton de la Fournaise volcano, they have retrieved the Rayleigh-wave group velocity distribution as a function of frequency and used this to derive a 3D S-wave velocity model of the interior of the volcano. In the past couple of years, the applications of direct surface-wave interferometry have expanded spectacularly. Without any claim of completeness, we mention Larose et al. (2005), Gerstoft et al. (2006), Kang and Shin (2006), Larose et al. (2006), Yao et al. (2006), Bensen et al. (2008), Gouédard et al. (2008a, 2008b), Liang and Langston (2008), Lin et al. (2008), Ma et al. (2008), Yao et al. (2008), Li et al. (2009), and Picozzi et al. (2009). The success of these applications is explained by the fact that surface waves are by far the strongest events in ambient seismic noise. In the next section, we show that the retrieval of reflected waves from ambient seismic noise is an order more difficult.

Direct surface-wave interferometry has an interesting link with early work by Aki $(1957,1965)$ and Toksöz (1964) on the spatial autocorrelation (SPAC) method. The SPAC method uses a circular array of seismometers plus a seismometer at the center of the circle. For a distribution of uncorrelated fundamental-mode Rayleigh waves propagating as plane waves in all directions, the spatial autocorrelation function obtained from the circular array reveals the local surface-wave velocity as a function of frequency and, subsequently, the local depth-dependent velocity profile. An important difference with the interferometry approach is that the distances between the receivers in the SPAC method are usually smaller than half a wavelength (Henstridge, 1979), making it a local method; whereas in direct-wave interferometry, the distances are assumed much larger than the wavelength because otherwise the stationary-phase arguments would not hold. More recent discussions on the SPAC method are given by Okada $(2003,2006)$ and Asten (2006). An interesting discussion on the relation between the SPAC method and seismic interferometry is given by Yokoi and Margaryan (2008).

\section{REFLECTED-WAVE INTERFEROMETRY}

\section{D analysis of reflected-wave interferometry}

The figure on the cover of Schuster's book on seismic interferometry (Schuster, 2009), reproduced in Figure 8, explains the basic principle of reflected-wave interferometry very well. Figure 8a shows a source in the subsurface that radiates a transient wave to the earth's surface, where it is received by a geophone. The trace contains the delayed source wavelet. Figure $8 \mathrm{~b}$ shows how the wave is reflected downward by the surface, reflected upward again by a scatterer in the subsurface, and received by a second geophone at the earth's surface. The trace contains the wavelet, which is further delayed due to the propagation along the additional path from receiver 1 via the scatterer to receiver 2. The propagation paths in Figure 8a and $b$ have the path from the subsurface source to the first receiver in common. By crosscorrelating the two traces (Schuster denotes this by $\otimes)$, the propagation along this common path is eliminated, leaving the path from receiver 1 via the scatterer to receiver 2 (Figure 8c). Hence, the result can be interpreted as a reflection experiment with a source at the position of the first geophone, of which the reflection response is received by the second geophone.

Let us see how this method deals with multiple reflections. To this end, we consider a configuration consisting of a homogeneous lossless layer, sandwiched between a free surface and a homogeneous lossless half-space (Figure 9a). An impulsive unit source in the lower half-space emits a vertically upward-propagating plane wave that reaches the surface after a time $t_{0}$. Because it was transmitted by a single interface on its way to the surface, the first arrival is given by $\tau \delta\left(t-t_{0}\right)$, where $\tau$ is the transmission coefficient of the interface (we use lowercase symbols for local transmission and reflection coefficients). This arrival is represented by the impulse at $t=t_{0}$ in Figure $9 \mathrm{~b}$. The wave is reflected downward by the free surface (reflection coefficient -1 ) and subsequently reflected upward by the interface (reflection coefficient $r$ ). Therefore, the next arrival reaching the surface is $-r \tau \delta\left(t-t_{0}-\Delta t\right)$, with $\Delta t=2 \Delta z / c$, where $\Delta z$ is the thickness of the first layer and $c$ its propagation velocity. Figure $9 \mathrm{~b}$ shows the total upgoing wavefield reaching the free surface - denoted as $T(t)$, where $T$ stands for the global transmission response. It consists of an infinite series of impulses with regular intervals $\Delta t$ (starting at $t_{0}$ ) and amplitudes $a_{0}=\tau, a_{1}=-r \tau, a_{2}=r^{2} \tau, a_{3}=$ $-r^{3} \tau$, etc.

Seismic interferometry for a vertically propagating plane wave reduces to evaluating the autocorrelation of the global transmission response, i.e., $T(t) * T(-t)$. We obtain the simplest result if we consider so-called power-flux normalized up- and downgoing waves (Frasier, 1970; Kennett et al., 1978; Ursin, 1983; Chapman, 1994). This means we define the local transmission coefficient $\tau$ as the square root of the product of the transmission coefficients for acoustic pressure and particle velocity. Hence, for an upgoing wave, $\tau$ $=\sqrt{(1-r)(1+r)}=\sqrt{1-r^{2}}$ (which is also the transmission coefficient for a downgoing wave). The autocorrelation for zero time lag is

$$
\begin{gathered}
\left(a_{0}^{2}+a_{1}^{2}+a_{2}^{2}+a_{3}^{2}+\cdots\right) \delta(t)=\tau^{2}\left(1+r^{2}+r^{4}+r^{6}\right. \\
+\cdots) \delta(t)=\tau^{2}\left(1-r^{2}\right)^{-1} \delta(t)=\delta(t) .
\end{gathered}
$$

This is represented by the impulse at $t=0$ in Figure $9 \mathrm{c}$. The autocorrelation for time lag $\Delta t$ is

$$
\begin{aligned}
\left(a_{1} a_{0}\right. & \left.+a_{2} a_{1}+a_{3} a_{2}+\cdots\right) \delta(t-\Delta t)=-r \tau^{2}\left(1+r^{2}+r^{4}\right. \\
& +\cdots) \delta(t-\Delta t)=-r \delta(t-\Delta t)
\end{aligned}
$$

which is represented by the impulse at $\Delta t$ in Figure $9 \mathrm{c}$. For time lags $2 \Delta t, 3 \Delta t$, etc., we obtain $r^{2} \delta(t-2 \Delta t),-r^{3} \delta(t-3 \Delta t)$, etc. Apart from an overall minus sign, these impulses together (except the one at $t=0$ ) represent the global reflection response $R(t)$ of a downgo-
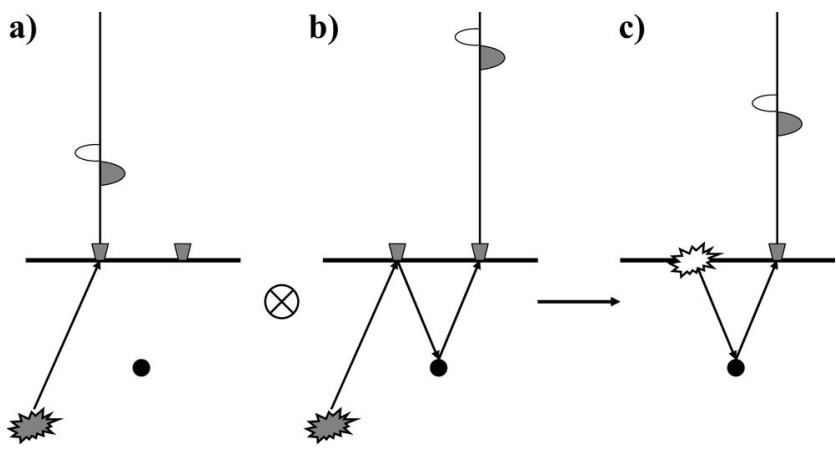

Figure 8. Basic principle of reflected-wave interferometry (Schuster, 2001, 2009). (a) A subsurface source emits a wave to the surface where it is received by a geophone. (b) A second geophone receives a reflected wave. (c) Crosscorrelation eliminates the propagation along the path from the source to the first geophone. The result is interpreted as the reflection response of a source at the position of the first geophone, observed by the second geophone. 
ing plane wave, illuminating the medium from the free surface. Consequently, the causal part of the autocorrelation is equal to $-R(t)$. Similarly, the acausal part is $-R(-t)$. Taking everything together, we have $T(t) * T(-t)=\delta(t)-R(t)-R(-t)$, or

$$
R(t)+R(-t)=\delta(t)-T(t) * T(-t) .
$$

Expression 8 shows that the global reflection response can be obtained from the autocorrelation of the global transmission response. This concept can be understood intuitively if one bears in mind that the reflection response, including all its multiples, is implicitly present in the coda of the transmission response (see Figure 9b). Note the analogy of equation 8 with the expression for direct-wave interferometry (equation 4). In both cases, the left-hand side is a superposition of a causal response and its time-reversed version. The main difference is that the right-hand side of equation 4 is a superposition of crosscorrelations of rightward- and leftward-propagating waves, which was necessary to get the time-symmetric response, whereas the right-hand side of equation 8 is a single autocorrelation. The free surface in Figure 9a acts as a mirror, which removes the requirement of having sources at both sides of the receivers to obtain a time-symmetric response.

It can easily be shown that equation 8 holds for arbitrary horizontally layered media. To this end, consider the configuration in Figure $9 \mathrm{~d}$. Here, the illuminating wavefield is an impulsive downgoing plane wave at the free surface (denoted by $\delta(t)$ in Figure $9 \mathrm{~d}$ ). The upgoing wave arriving at the free surface is the global reflection response $R(t)$, which is reflected downward by the free surface with reflection coefficient -1 . Accordingly, the total downgoing wavefield just below the surface is $D(t)=\delta(t)-R(t)$ and the total upgoing wavefield is $U(t)=R(t)$. The total downgoing wavefield below the lowest interface is given by the global transmission response $T(t)$. We assume again that the downgoing and upgoing waves are flux

a)

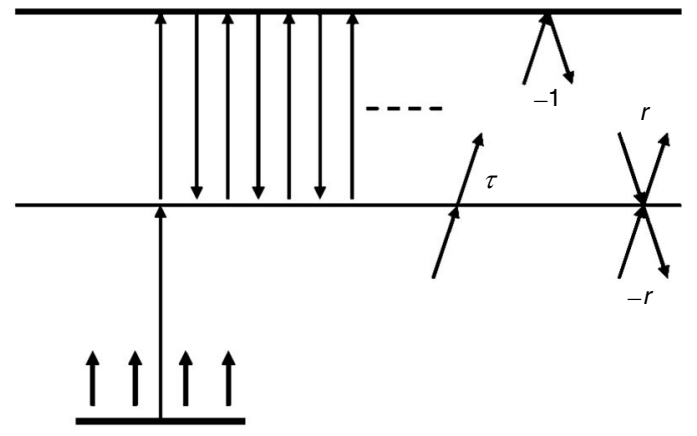

b)

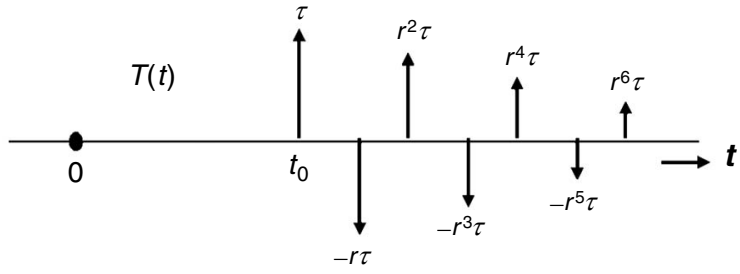

normalized. Hence, the global transmission response of the downgoing plane wave source at the free surface is equal to that of an upgoing plane-wave source below the lowest interface (Frasier, 1970).

Because we consider a lossless medium, we can use the principle of power conservation to derive a relation between the wavefields at the top and the bottom of the configuration. The power flux is most easily defined in the frequency domain. To this end, we define the Fourier transform of a time-dependent function as

$$
\hat{f}(\omega)=\int_{-\infty}^{\infty} f(t) \exp (-j \omega t) \mathrm{d} t,
$$

where $\omega$ is the angular frequency and $j$ the imaginary unit. The net power flux just below the free surface is given by

$$
\hat{D} \hat{D}^{*}-\hat{U} \hat{U}^{*}=(1-\hat{R})\left(1-\hat{R}^{*}\right)-\hat{R} \hat{R}^{*}=1-\hat{R}-\hat{R}^{*}
$$

where the superscript asterisk denotes complex conjugation. The net power flux is independent of depth, so the right-hand side of equation 10 is equal to the net power flux in the lower half-space $\hat{T} \hat{T}^{*}$. Thus, $1-\hat{R}-\hat{R}^{*}=\hat{T} \hat{T}^{*}$, or

$$
\hat{R}+\hat{R}^{*}=1-\hat{T} \hat{T}^{*} .
$$

Complex conjugation in the frequency domain corresponds to time reversal in the time domain, so the inverse Fourier transform of this equation again gives equation 8 , which has now been proven to hold for arbitrarily layered media.

The central assumption in this derivation is the conservation of acoustic power, which of course only holds in lossless media. We assumed in our discussions of direct-wave interferometry that the medium was lossless; but in the present derivation, the essence of this

c)

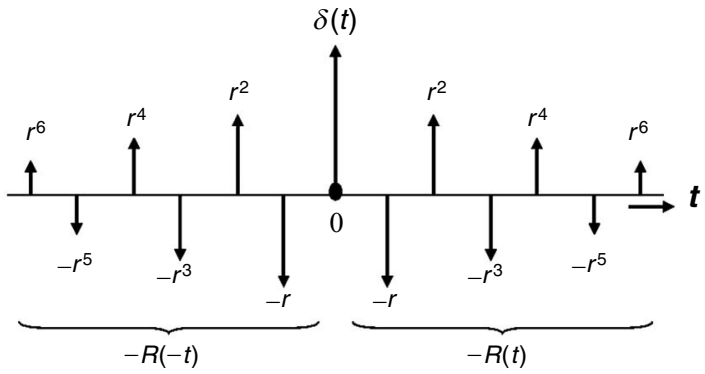

d)

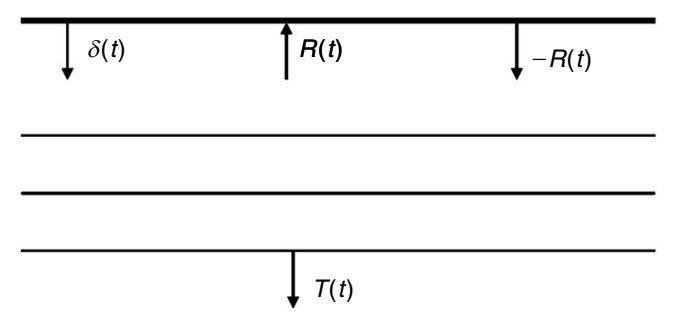

Figure 9. From transmission to reflection response (1D). (a) Simple layered medium with an upgoing plane wave radiated by a source in the lower half-space. (b) The transmission response $T(t)$ observed at the free surface. (c) The autocorrelation $T(t) * T(-t)$. The causal part is, apart from a minus sign, the reflection response $R(t)$. (d) Configuration used to derive the same relation for an arbitrarily layered medium. 
assumption has become manifest. Most approaches to seismic interferometry rely on the assumption that the medium is lossless. In Part 2 of this tutorial, we also encounter approaches that account for losses or that use the essence of this assumption to estimate loss parameters.

We should note here that equation 8 for arbitrarily layered media was derived more than 40 years ago by Jon Claerbout at Stanford University (Claerbout, 1968). His expression looks slightly different because he did not use flux normalization. For his derivation, he used a recursive method introduced by Thomson (1950), Haskell (1953), and others. Later, he proposed the shorter derivation using energy conservation (Claerbout, 2000). Frasier (1970) generalizes Claerbout's result for obliquely propagating plane $\mathrm{P}$ - and $\mathrm{SV}$-waves in a horizontally layered elastic medium.

Analogous to equations 5 and 7, equation 8 can be modified for transient or noise signals. For example, let $u(t)=T(t) * N(t)$ be the upgoing wavefield at the surface, with $N(t)$ representing the noise signal emitted by the source in the lower half-space. Then we obtain from equation 8

$$
\{R(t)+R(-t)\} * S_{N}(t)=S_{N}(t)-\langle u(t) * u(-t)\rangle,
$$

where $S_{N}(t)$ is the autocorrelation of the noise. Equation 12 shows that the autocorrelation of passive noise measurements gives the reflection response of a transient source at the surface. Quite remarkable indeed! Again, the position of the actual source does not need to be known, but it should lie below the lowest interface. In the next section, we show that the latter assumption can be relaxed in $2 \mathrm{D}$ and 3D configurations.

Early applications of equation 12, some more successful than others, are discussed by Baskir and Weller (1975), Scherbaum (1987a, 1987b), Cole (1995), Daneshvar et al. (1995), and Poletto and Petronio $(2003,2006)$.

\section{D and 3D analysis of reflected-wave interferometry}

Claerbout conjectured for the 2D and 3D situation that "by crosscorrelating noise traces recorded at two locations on the surface, we can construct the wavefield that would be recorded at one of the locations if there was a source at the other" (citation is from Rickett and Claerbout [1999], but the conjecture is also mentioned by Cole [1995]). This statement could be applied literally to direct-wave interferometry, as discussed in a previous section, but Claerbout's conjecture concerns reflected-wave interferometry. Of course, this terminology was not used by these authors, and the links between direct-wave and reflected-wave interferometry were discovered several years later. Duvall et al. (1993) and Rickett and Claerbout (1999) applied crosscorrelations to noise observations at the surface of the sun and were able to retrieve helioseismological shot records.

Claerbout's 1D relation (equation 8) and his conjecture for the 3D situation inspired Jerry Schuster at the University of Utah. During a sabbatical in 2000 at Stanford University, Schuster analyzed the conjecture by the method of stationary phase. Let us briefly review his line of thought (Schuster, 2001; Schuster et al., 2004; Schuster and Zhou, 2006). First, consider again the configuration shown in Figure 8. It was implicitly assumed that the first geophone is located precisely at the specular reflection point of the drawn ray in Figure $8 \mathrm{~b}$. As a consequence, the ray in Figure 8a coincides with the first branch of the ray in Figure 8b; so in a 1D crosscorrelation process, the traveltime along this ray cancels, which leaves the traveltime of the reflection response. In practice, the source position and hence the position of the specular reflection point are unknown. However, when there are multiple (unknown) sources in the subsurface, it is again possible to extract the reflection response.

To see this, consider the situation depicted in Figure 10a, in which there are multiple sources buried in the subsurface. The ray that leaves the source at $x_{1, S}=-300 \mathrm{~m}$ reflects at $\mathbf{x}_{A}$ (the position of the first geophone) on its way to the scatterer at $\mathbf{x}_{D}$ and the second geophone at $\mathbf{x}_{B}$; this is the specular ray. The rays leaving the other sources have their specular reflection points left and right from $\mathbf{x}_{A}$ (the solid rays in Figure 10a). The direct arrivals at $\mathbf{x}_{A}$ follow the dashed paths and do not coincide with the solid rays, except for the source at $x_{1, S}=-300 \mathrm{~m}$. For each of the sources, we crosscorrelate the direct arrival at $\mathbf{x}_{A}$ with the scattered wave recorded at $\mathbf{x}_{B}$. This gives the correlation gather shown in Figure 10b, in which the horizontal axis denotes the source coordinate $x_{1, S}$. The trace at $x_{1, S}=-300 \mathrm{~m}$ shows an impulse (indicated by the vertical arrow) at $t_{A B}$, which is the traveltime from $\mathbf{x}_{A}$ via the scatterer to $\mathbf{x}_{B}$. The impulses in the surrounding traces arrive before $t_{A B}$.

If we sum the traces for all $x_{1, S}$, the main contribution comes from an area (the Fresnel zone, indicated by the dashed lines) around the point $x_{1, S}=-300 \mathrm{~m}$ where the traveltime curve is stationary (indicated by the vertical arrow); the other contributions cancel. Hence, the sum of the correlations (Figure 10c) contains an impulse at $t_{A B}$ and can be interpreted as the reflection response that would be mea-

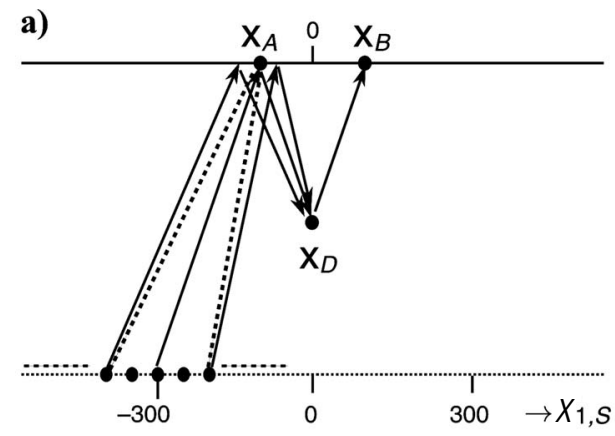

b)

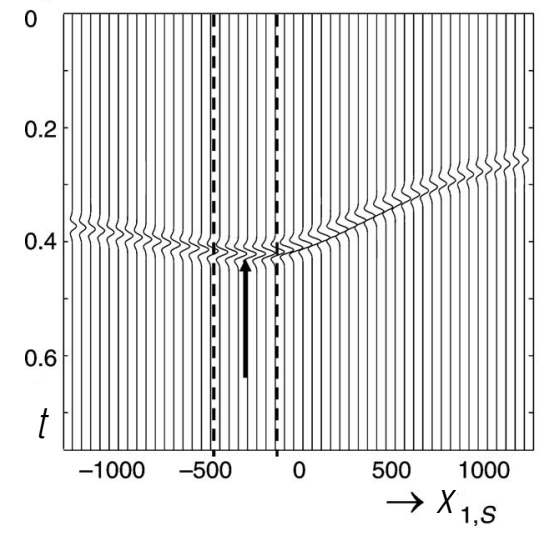

c)

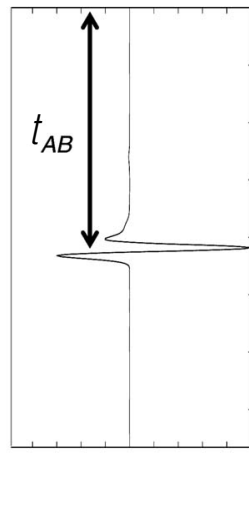

Figure 10. Basic principle of reflected-wave interferometry revisited. (a) Configuration with multiple sources in the subsurface. Only the ray emitted by the source at $x_{1, S}=-300 \mathrm{~m}$ has its specular reflection point at one of the geophone positions. (b) Crosscorrelations of the responses at $\mathbf{x}_{A}$ and $\mathbf{x}_{B}$ as a function of the source coordinate $x_{1, S}$. The traveltime curve connecting these events is stationary at $x_{1, S}=-300 \mathrm{~m}$. The thick dashed lines indicate the Fresnel zone. (c) The sum of the correlations in (b). This is interpreted as the reflection response of a source at $\mathbf{x}_{A}$ observed by a receiver at $\mathbf{x}_{B}$. 
sured at $\mathbf{x}_{B}$ if there were a source at $\mathbf{x}_{A}$. In other words, the source has been repositioned from its unknown position at depth to a known position $\mathbf{x}_{A}$ at the surface. Note that this procedure works for any $\mathbf{x}_{A}$ and $\mathbf{x}_{B}$ as long as the array of sources contains a source that emits a specular ray via $\mathbf{x}_{A}$ and the scatterer to $\mathbf{x}_{B}$. In Appendix A, we give a simple proof that the stationary point of the traveltime curve in a correlation gather corresponds to the source from which the rays to $\mathbf{x}_{A}$ and $\mathbf{x}_{B}$ leave in the same direction.

This example shows that it is possible to reposition (or redatum) sources without knowing the velocity model and the position of the original sources. In exploration geophysics, redatuming is known as a process that brings sources and/or receivers from the acquisition level to another depth level, using extrapolation operators based on a macro velocity model (Berryhill, 1979, 1984). In seismic interferometry, as illustrated in Figure 10, the extrapolation operator comes directly from the data (in this example, the observed direct wave at $\mathbf{x}_{A}$.

In the years following his sabbatical, Schuster showed that the interferometric redatuming concept, indicated in Figure 10, can be applied to a wide range of configurations (mostly for controlled-source data). His work inspired many other researchers to develop interferometric methods for exploration geophysics. For example, VSP data can be transformed into crosswell data (Minato et al., 2007) or into single-well reflection profiles to improve salt-flank delineation and imaging (Willis et al., 2006; Xiao et al., 2006; Hornby and Yu, 2007;
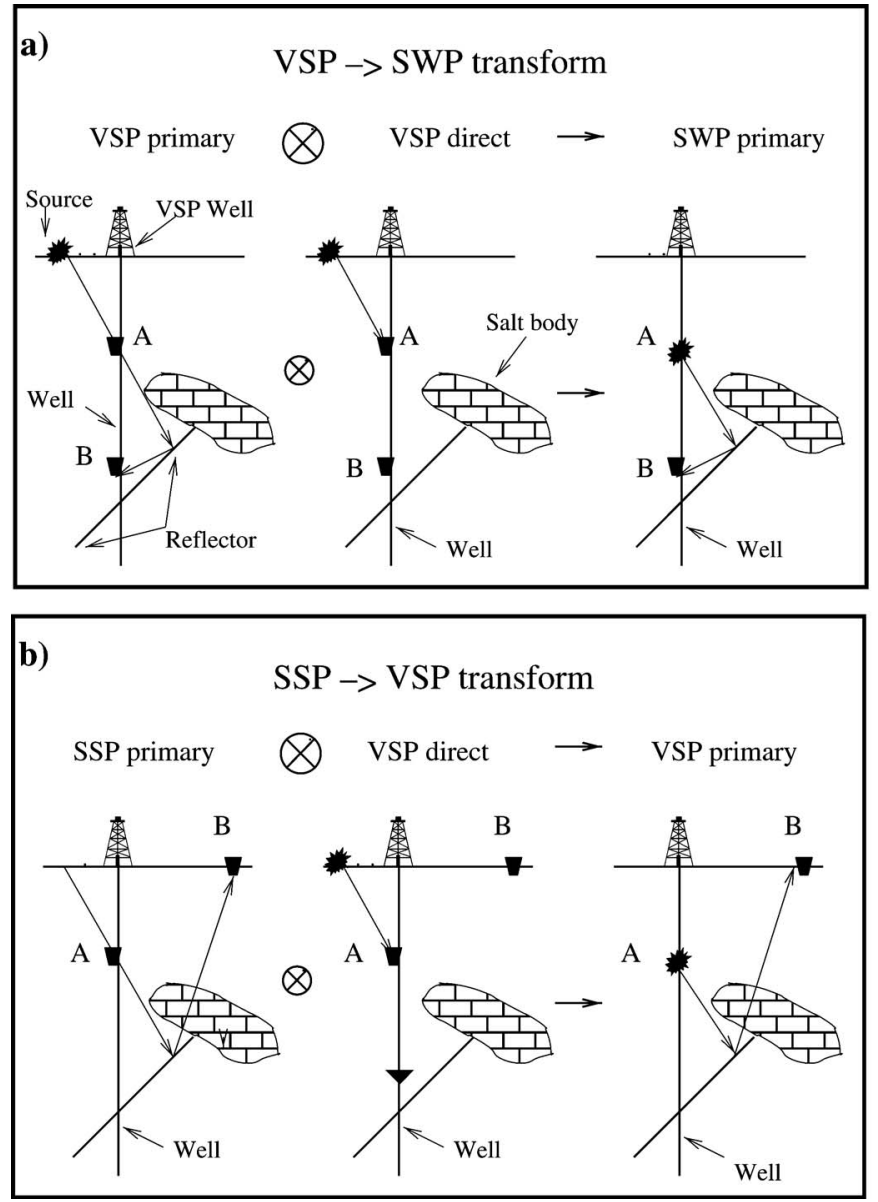

Lu et al., 2008). Interferometry can be used to turn multiples in VSP data into primaries and in this way enlarge the illuminated area ( $\mathrm{Yu}$ and Schuster, 2006; He et al., 2007; Jiang et al., 2007). Surface multiples can be turned into primaries at the position of missing traces (Wang et al., 2009). Crosscorrelation of refracted waves gives virtual refractions that can be used for improved estimation of the subsurface parameters (Dong et al., 2006b; Mikesell et al., 2009). Surface waves can be predicted by interferometry and subsequently subtracted from exploration seismic data (Curtis et al., 2006; Dong et al., 2006a; Halliday et al., 2007, 2010; Xue et al., 2009). In his recent book, Schuster (2009) systematically discusses all possible interferometric transformations between surface data, VSP data, single-well profiles, and crosswell data. Figure 11 shows some examples. Another approach to interferometric redatuming of controlled-source data, known as the virtual-source method (Bakulin and Calvert, 2004, 2006), is discussed in Part 2 of this paper.

The example discussed in Figure 10 deals with primary reflections and therefore confirms Claerbout's conjecture only partly. The $1 \mathrm{D}$ analysis in the previous section showed that not only primary reflections but also all multiples are recovered from the autocorrelation of the transmission response. Claerbout's conjecture for the 3D situation can be proven along similar lines. Instead of using the principle of power conservation, a so-called power reciprocity theorem is used as the starting point. In general, an acoustic reciprocity theorem formulates a relation between two acoustic states (de Hoop, c)

$$
\text { SSP } \rightarrow \text { SSP transform }
$$

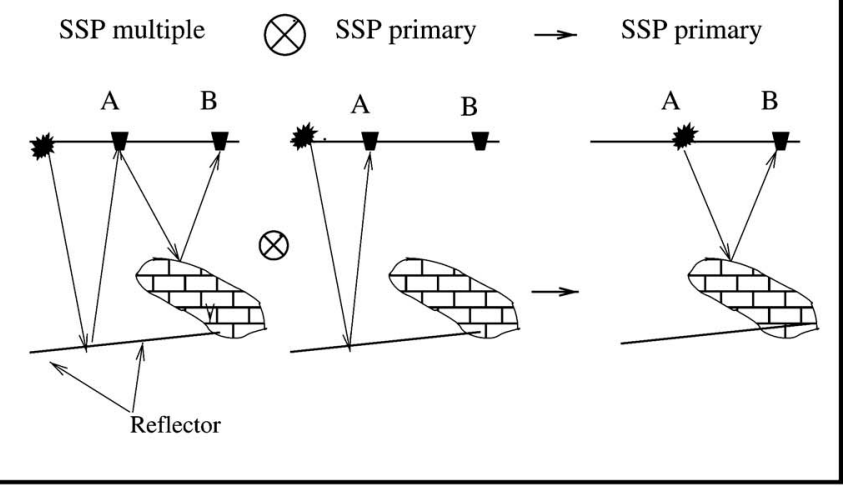

d)

$$
\text { VSP } \rightarrow \text { Xwell transform }
$$

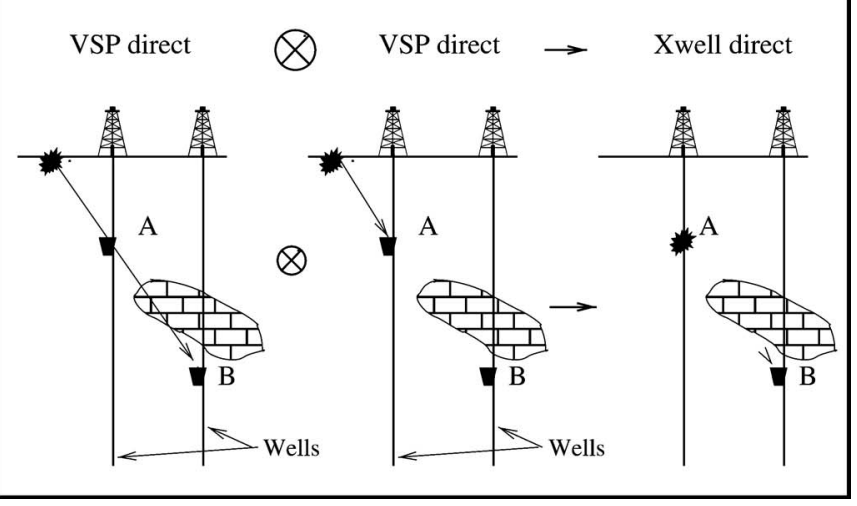

Figure 11. Some examples of interferometric redatuming (Schuster, 2009). Each diagram shows that crosscorrelation of the trace recorded at $A$ with the one at $B$ and summing over source locations leads to the response of a source at $A$, closer to the target than the original sources. 
1988; Fokkema and van den Berg, 1993). One can distinguish between convolution and correlation reciprocity theorems. The theorems of the correlation type reduce to power-conservation laws when the two states are chosen identical, which is why they are also called power reciprocity theorems. Because reflection and transmission responses are defined for downgoing and upgoing waves, for the proof of Claerbout's conjecture we make use of a correlation reciprocity theorem for (flux-normalized) one-way wavefields (Wapenaar and Grimbergen, 1996).

Consider the configuration in Figure 12a. An arbitrary inhomogeneous lossless medium is sandwiched between a free surface and a homogeneous lower half-space. Impulsive sources are distributed along a horizontal plane in this lower half-space. For this configuration, we derive (Wapenaar et al., 2002, 2004)

$$
\begin{aligned}
& R\left(\mathbf{x}_{B}, \mathbf{x}_{A}, t\right)+R\left(\mathbf{x}_{B}, \mathbf{x}_{A},-t\right) \\
& \quad \approx \delta\left(\mathbf{x}_{H, B}-\mathbf{x}_{H, A}\right) \delta(t)-\sum_{i} T\left(\mathbf{x}_{B}, \mathbf{x}_{S}^{(i)}, t\right) * T\left(\mathbf{x}_{A}, \mathbf{x}_{S}^{(i)},-t\right) .
\end{aligned}
$$

Here, $\mathbf{x}_{H, A}$ and $\mathbf{x}_{H, B}$ denote the horizontal components of $\mathbf{x}_{A}$ and $\mathbf{x}_{B}$, respectively; $T\left(\mathbf{x}_{A(B)}, \mathbf{x}_{S}^{(i)}, t\right)$ is the upgoing transmission response of an impulsive point source at $\mathbf{x}_{S}^{(i)}$ in the subsurface, observed at $\mathbf{x}_{A(B)}$ at the free surface. Its coda includes all surface-related and internal multiple reflections (only a few rays are shown in Figure 12a). The right-hand side of equation 13 involves a crosscorrelation of transmission responses at $\mathbf{x}_{A}$ and $\mathbf{x}_{B}$ for each source $\mathbf{x}_{S}^{(i)}$, followed by a summation over all source positions. The time-symmetric response on the left-hand side is the reflection response that would be recorded at $\mathbf{x}_{B}$ if there were a source at $\mathbf{x}_{A}$, plus its time-reversed version. The main approximation is the negligence of evanescent waves. Apart from that, the retrieved reflection response $R\left(\mathbf{x}_{B}, \mathbf{x}_{A}, t\right)$ contains all primary, surface-related, and internal multiple reflections. They are unraveled by equation 13 from the coda of the transmission responses.

When the impulsive sources are replaced by uncorrelated noise sources, then the responses at $\mathbf{x}_{A}$ and $\mathbf{x}_{B}$ are given by $u\left(\mathbf{x}_{A}, t\right)$ $=\Sigma_{i} T\left(\mathbf{x}_{A}, \mathbf{x}_{S}^{(i)}, t\right) * N_{i}(t)$ and $u\left(\mathbf{x}_{B}, t\right)=\Sigma_{j} T\left(\mathbf{x}_{B}, \mathbf{x}_{S}^{(j)}, t\right) * N_{j}(t)$ (see Figure $12 \mathrm{~b}$, where each dashed ray represents a complete transmission response). Using a derivation similar to the one that transforms equation 4 into equation 7 , we obtain from equation 13

$$
\begin{aligned}
& \left\{R\left(\mathbf{x}_{B}, \mathbf{x}_{A}, t\right)+R\left(\mathbf{x}_{B}, \mathbf{x}_{A},-t\right)\right\} * S_{N}(t) \\
& \quad \approx \delta\left(\mathbf{x}_{H, B}-\mathbf{x}_{H, A}\right) S_{N}(t)-\left\langle u\left(\mathbf{x}_{B}, t\right) * u\left(\mathbf{x}_{A},-t\right)\right\rangle,
\end{aligned}
$$

where $S_{N}(t)$ is the autocorrelation of the noise.

Equation 14 shows that the direct crosscorrelation of passive noise measurements gives the reflection response of a transient source at the free surface. Although equations 13 and 14 are derived for a situation in which the sources at $\mathbf{x}_{S}^{(i)}$ lie at the same depth (Figure 12a), these equations remain approximately valid when the depths are randomly distributed (as in Figure 12b) because in the crosscorrelation process, only the time difference matters (we used a similar reasoning for direct-wave interferometry to explain why the traveltime curves in Figure 6d remain smooth). Moreover, despite the initial assumption that the medium is homogeneous below the sources, Draganov et al. (2004) show with numerical examples that the randomness of the source depths helps to suppress nonphysical ghosts related to reflectors below the sources, whereas the physical response of these deeper reflectors shows up correctly in $R\left(\mathbf{x}_{B}, \mathbf{x}_{A}, t\right)$. This has also been explained with theoretical arguments (Wapenaar and Fokkema, 2006).

Equations 13 and 14 are used by various authors to turn ambient seismic noise into virtual exploration seismic reflection data (Draganov et al., 2006, 2007, 2009; Hohl and Mateeva, 2006; Torii et al., 2007). Interestingly, the teleseismic community has recognized independently that the coda of transmission responses from distant sources contains reflection information which can be used to image the earth's crust (Bostock et al., 2001; Rondenay et al., 2001; Shragge et al., 2001, 2006; Mercier et al., 2006). The link between teleseismic coda imaging and seismic interferometry is exploited by Kumar and Bostock (2006), Nowack et al. (2006), Chaput and Bostock (2007), and Tonegawa et al. (2009).

We conclude this section with an example of retrieving virtual exploration seismic reflection data from ambient noise, recorded by Shell in a desert area near Ajdābiya, Libya. Figure 13a shows $10 \mathrm{~s}$ of noise, arbitrarily selected from 11 hours of noise, recorded along a $20-\mathrm{km}$ line. Each receiver channel represents a group of 48 verticalcomponent geophones, designed to suppress surface waves. Nevertheless, the main events in Figure 13a are parts of the surface waves that fell outside the suppression band of the geophone groups; these surface waves were caused by traffic on a road intersecting the line at $x_{1}=14 \mathrm{~km}$. Band-pass and frequency-wavenumber $(f-k)$ filtering were used to suppress the surface waves further (Figure 13b).
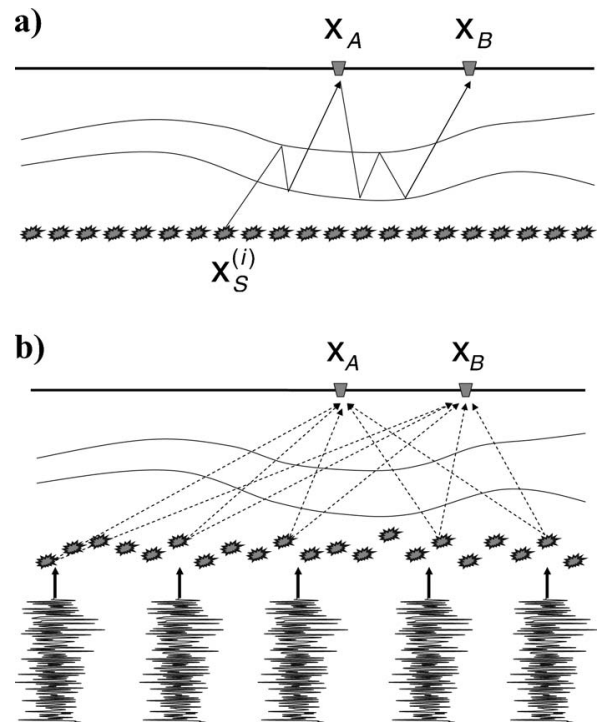

Figure 12. From transmission to reflection response (3D). (a) Arbitrary inhomogeneous lossless medium, with sources in the homogeneous lower half-space and receivers at $\mathbf{x}_{A}$ and $\mathbf{x}_{B}$ at the free surface. According to equation 13, the reflection response $R\left(\mathbf{x}_{B}, \mathbf{x}_{A}, t\right)$, implicitly present in the coda of the transmission response, is retrieved by crosscorrelating transmission responses observed at $\mathbf{x}_{A}$ and $\mathbf{x}_{B}$ and summing over the sources. (b) When the sources are simultaneously acting mutually uncorrelated noise sources, the observed responses at $\mathbf{x}_{A}$ and $\mathbf{x}_{B}$ are each a superposition of transmission responses. According to equation 14, the reflection response $R\left(\mathbf{x}_{B}, \mathbf{x}_{A}, t\right)$ is now retrieved from the direct crosscorrelation of the observations at $\mathbf{x}_{A}$ and $\mathbf{x}_{B}$. 
We use equation 14 to retrieve the reflection response. Strictly speaking, equation 14 requires decomposition of the filtered geophone data of Figure 13b into the upgoing transmission response. In the acoustic approximation, decomposition mainly involves the application of an angle-dependent amplitude filter. Because it is very difficult to obtain true amplitude responses from ambient noise anyway, the decomposition step is skipped. Using equation 14, with $\mathbf{x}_{A}$ fixed $\left(x_{1, A}=1 \mathrm{~km}\right)$ and $\mathbf{x}_{B}$ chosen variable $\left(x_{1, B}=0, \ldots, 4 \mathrm{~km}\right)$, we retrieve a seismic shot record $R\left(\mathbf{x}_{B}, \mathbf{x}_{A}, t\right)$ from the noise, of which the first $2.5 \mathrm{~s}$ are shown in Figure 14a. The red star at $x_{1, B}=x_{1, A}=1 \mathrm{~km}$ denotes the position of the virtual source. An active seismic reflection experiment, carried out with the source at the same position, is shown in Figure 14b. Particularly in the red areas, the reflections retrieved from the ambient noise (Figure 14a) correspond quite well with those in the active shot gather (Figure 14b). For more details about this experiment as well as a pseudo-3D reflection image obtained from the ambient noise, see Draganov et al. (2009).

\section{CONCLUSIONS}

We have discussed the basic principles of seismic interferometry in a heuristic way. We have shown that, whether we consider controlled-source or passive interferometry, virtual sources are created at positions where there are only receivers. Of course, no new information is generated by interferometry, but information hidden in noise or in a complex scattering coda is reorganized into easily interpretable responses that can be further processed by standard tomographic inversion or reflection-imaging methodologies. The main strength is that this information unraveling requires no knowledge of the subsurface medium parameters nor of the positions or timing of the actual sources. Moreover, the processing consists of simple crosscorrelations and is almost entirely data driven.

In Part 2, we discuss the relation between interferometry and time-reversed acoustics, review a mathematically sound derivation, and indicate recent and new advances. a)

$$
\rightarrow x_{1}(\mathrm{~km})
$$

$\begin{array}{lllllllllll}0 & 2 & 4 & 6 & 8 & 10 & 12 & 14 & 16 & 18 & 20\end{array}$

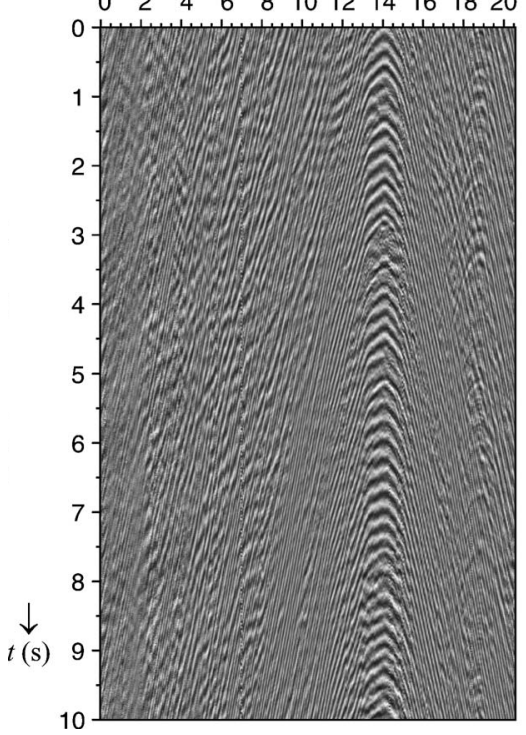

b)

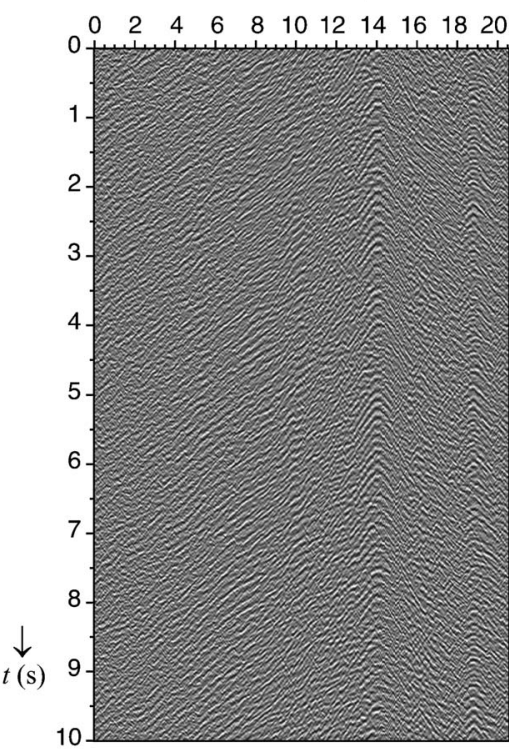

Figure 13. (a) Ten seconds of ambient noise, arbitrarily selected from 11 hours of noise, recorded in a desert area near Ajdābiya, Libya. The main events are remnants of surface waves caused by traffic at $x_{1}=14 \mathrm{~km}$. (b) The same noise window after further suppression of the surface waves.

a)

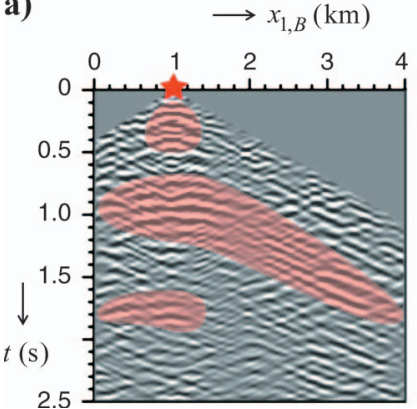

b)

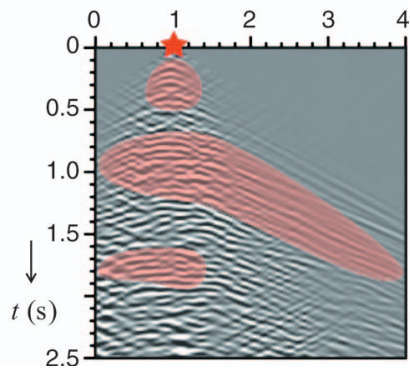

Figure 14. (a) Reflection response (shot record) obtained by crosscorrelating 11 hours of ambient noise (Draganov et al., 2009). (b) For comparison, an active shot record measured at the same location.

\section{ACKNOWLEDGMENTS}

This work is supported by the Netherlands Research Centre for Integrated Solid Earth Science (ISES), the Dutch Technology Foundation (STW) (grant VENI.08115), and by the U. S. National Science Foundation (NSF) (grant EAS-0609595). We thank associate editor Sven Treitel and reviewers Jerry Schuster, Kasper van Wijk, and Mark Willis for their valuable comments and suggestions, which improved this paper. We are grateful to Jerry Schuster, Fan-Chi Lin, Mike Ritzwoller, and Wiley-Blackwell (publisher of Geophysical Journal International) for Figures 11 and 7. Last but not least, we thank the Libyan National Oil Company for permission to publish the Ajdabeya data results and Shell in Libya for collecting and making available the passive data.

\section{APPENDIX A}

\section{STATIONARY-PHASE ANALYSIS}

We give a simple proof that the stationary point of the traveltime curve in a correlation gather corresponds to the source from which the rays to the receivers at $\mathbf{x}_{A}$ and $\mathbf{x}_{B}$ leave in the same direction. Consider two rays $A$ and $B$ that propagate from an arbitrary source point to the two receivers (Figure A-1). This propagation may be direct, or it may involve bounces off reflectors or scatterers; the fate of these rays is irrelevant for the argument presented here. The sources involved in interferometry are located on the surface, indicated by the dashed line in Figure A-1. This surface, which need not be planar, is in three dimensions parameterized by two orthogonal coordinates $q_{1}$ and $q_{2}$. We first keep $q_{2}$ fixed and consider only variations in $q_{1}$.

The traveltime from a given source to the receiver at $\mathbf{x}_{A}$ is denoted by $t_{A}$, and the traveltime from that source to the receiver at $\mathbf{x}_{B}$ is denoted by $t_{B}$. These traveltimes are, in general, functions of the source position $q_{1}$. In seismic interferometry, the traveltimes of the signals 


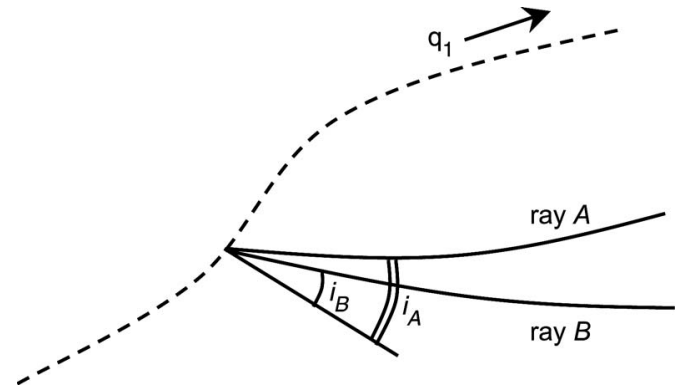

Figure A-1. Two rays, $A$ and $B$, that propagate from a common point on the surface with sources (dashed line) and their take-off angles at this source surface.

that are crosscorrelated are subtracted. This means that the traveltime $t_{\text {corr }}$ of the crosscorrelation for a given source position is

$$
t_{\text {corr }}\left(q_{1}\right)=t_{B}\left(q_{1}\right)-t_{A}\left(q_{1}\right) .
$$

The condition that the traveltime is stationary means that

$$
\frac{\partial t_{\mathrm{corr}}\left(q_{1}\right)}{\partial q_{1}}=\frac{\partial t_{B}\left(q_{1}\right)}{\partial q_{1}}-\frac{\partial t_{A}\left(q_{1}\right)}{\partial q_{1}}=0 .
$$

A standard derivation (Aki and Richards, 1980) relates the slowness along the surface to the take-off angle

$$
\frac{\partial t_{A}\left(q_{1}\right)}{\partial q_{1}}=\frac{\sin i_{A}}{c},
$$

with $c$ the propagation velocity. A similar expression holds for $t_{B}$. Inserting this in equation A-2 implies that, at the stationary point,

$$
i_{A}=i_{B},
$$

which means the rays take off in the same direction.

This reasoning is applicable to variations in the source coordinate $q_{1}$. The same reasoning applies to variations with the orthogonal source coordinate $q_{2}$. The rays take off in the same direction as measured in two orthogonal planes; hence, the rays have the same direction in three dimensions. Therefore, the rays radiating from the stationary source position are parallel.

\section{REFERENCES}

Aki, K., 1957, Space and time spectra of stationary stochastic waves, with special reference to micro-tremors: Bulletin of the Earthquake Research Institute, 35, 415-457.

- 1965, A note on the use of microseisms in determining the shallow structures of the earth's crust: Geophysics, 30, 665-666.

Aki, K., and P. G. Richards, 1980, Quantitative seismology, vol. 1: W. H. Freeman \& Co.

Asten M. W., 2006, On bias and noise in passive seismic data from finite circular array data processed using SPAC methods: Geophysics, 71, no. 6 , V153-V162.

Bakulin, A., and R. Calvert, 2004, Virtual source: New method for imaging and 4D below complex overburden: 74th Annual International Meeting, SEG, Expanded Abstracts, 2477-2480.

, 2006, The virtual source method: Theory and case study: Geophysics, 71, no. 4, SI139-SI150.

Baskir, E., and C. E. Weller, 1975, Sourceless reflection seismic exploration (abstract): Geophysics, 40, 158-159.

Bensen, G. D., M. H. Ritzwoller, M. P. Barmin, A. L. Levshin, F. Lin, M. P. Moschetti, N. M. Shapiro, and Y. Yang, 2007, Processing seismic ambient noise data to obtain reliable broad-band surface wave dispersion measurements: Geophysical Journal International, 169, 1239-1260.

Bensen, G. D., M. H. Ritzwoller, and N. M. Shapiro, 2008, Broadband ambi- ent noise surface wave tomography across the United States: Journal of Geophysical Research — Solid Earth, 113, B05306-1-B05306-21.

Berryhill, J. R., 1979, Wave-equation datuming: Geophysics, 44, 13291344.

- 1984, Wave-equation datuming before stack: Geophysics, 49, 2064 2066.

Bostock, M. G., S. Rondenay, and J. Shragge, 2001, Multiparameter two-dimensional inversion of scattered teleseismic body waves. 1 . Theory for oblique incidence: Journal of Geophysical Research, 106, 30771-30782.

Brenguier, F., N. M. Shapiro, M. Campillo, A. Nercessian, and V. Ferrazzini, 2007, 3-D surface wave tomography of the Piton de la Fournaise volcano using seismic correlations: Geophysical Research Letters, 34, L02305-1L02305-5.

Brooks, L. A., and P. Gerstoft, 2007, Ocean acoustic interferometry: Journal of the Acoustical Society of America, 121, 3377-3385.

Callen, H. B., and T. A. Welton, 1951, Irreversibility and generalized noise: Physical Review, 83, 34-40.

Campillo, M., and A. Paul, 2003, Long-range correlations in the diffuse seismic coda: Science, 299, 547-549.

Challis, L., and F. Sheard, 2003, The Green of Green functions: Physics Today, 56, 41-46.

Chapman, C. H., 1994, Reflection/transmission coefficients reciprocities in anisotropic media: Geophysical Journal International, 116, 498-501.

Chaput, J. A., and M. G. Bostock, 2007, Seismic interferometry using nonvolcanic tremor in Cascadia: Geophysical Research Letters, 34, L073041-L07304-5.

Claerbout, J. F., 1968, Synthesis of a layered medium from its acoustic transmission response: Geophysics, 33, 264-269.

- 2000, Introduction to acoustic daylight imaging, http:// sepwww.stanford.edu/data/media/public/sep//jon/eqcor/index.html, accessed 21 May 2010.

Cole, S., 1995, Passive seismic and drill-bit experiments using 2-D arrays: Ph.D. dissertation, Stanford University.

Curtis, A., P. Gerstoft, H. Sato, R. Snieder, and K. Wapenaar, 2006, Seismic interferometry - Turning noise into signal: The Leading Edge, 25, 1082-1092.

Daneshvar, M. R., C. S. Clay, and M. K. Savage, 1995, Passive seismic imaging using microearthquakes: Geophysics, 60, 1178-1186.

de Hoop, A. T., 1988, Time-domain reciprocity theorems for acoustic wave fields in fluids with relaxation: Journal of the Acoustical Society of America, 84, 1877-1882.

Dong, S., R. He, and G. T. Schuster, 2006a, Interferometric prediction and least squares subtraction of surface waves: 76th Annual International Meeting, SEG, Expanded Abstracts, 2783-2786.

Dong, S., J. Sheng, and G. T. Schuster, 2006b, Theory and practice of refraction interferometry: 76th Annual International Meeting, SEG, Expanded Abstracts, 3021-3025.

Draganov, D., X. Campman, J. Thorbecke, A. Verdel, and K. Wapenaar, 2009, Reflection images from ambient seismic noise: Geophysics, 74, no. 5, A63-A67.

Draganov, D., K. Wapenaar, W. Mulder, and J. Singer, 2006, Seismic interferometry on background-noise field data: 76th Annual International Meeting, SEG, Expanded Abstracts, 590-593.

Draganov, D., K. Wapenaar, W. Mulder, J. Singer, and A. Verdel, 2007, Retrieval of reflections from seismic background-noise measurements: Geophysical Research Letters, 34, L04305-1-L04305-4.

Draganov, D., K. Wapenaar, and J. Thorbecke, 2004, Passive seismic imaging in the presence of white noise sources: The Leading Edge, 23, no. 9 , 889-892.

Duvall, T. L., S. M. Jefferies, J. W. Harvey, and M. A. Pomerantz, 1993, Time-distance helioseismology: Nature, 362, 430-432.

Fan, Y., and R. Snieder, 2009, Required source distribution for interferometry of waves and diffusive fields: Geophysical Journal International, 179, $1232-1244$

Fokkema, J. T., and P. M. van den Berg, 1993, Seismic applications of acoustic reciprocity: Elsevier Scientific Publ. Co., Inc.

Frasier, C. W., 1970, Discrete time solution of plane P-SV waves in a plane layered medium: Geophysics, 35, 197-219.

Gerstoft, P., K. G. Sabra, P. Roux, W. A. Kuperman, and M. C. Fehler, 2006 Green's functions extraction and surface-wave tomography from microseisms in southern California: Geophysics, 71, no. 4, SI23-SI31.

Godin, O. A., 2007, Emergence of the acoustic Green's function from thermal noise: Journal of the Acoustical Society of America, 121, no. 2, EL96EL102

Gouédard, P., P. Roux, M. Campillo, and A. Verdel, 2008a, Convergence of the two-point correlation function toward the Green's function in the context of a seismic-prospecting data set: Geophysics, 73, no. 6, V47-V53.

Gouédard, P., L. Stehly, F. Brenguier, M. Campillo, Y. Colin de Verdière, E Larose, L. Margerin, P. Roux, F. J. Sánchez-Sesma, N. M. Shapiro, and R. L. Weaver, 2008b, Cross-correlation of random fields: Mathematical approach and applications: Geophysical Prospecting, 56, 375-393.

Green, G., 1828, An essay on the application of mathematical analysis to the 
theories of electricity and magnetism: Privately published.

Halliday, D. F., A. Curtis, J. O. A. Robertsson, and D.-J. van Manen, 2007, Interferometric surface-wave isolation and removal: Geophysics, 72, no. 5, A69-A73.

Halliday, D. F., A. Curtis, P. Vermeer, C. Strobbia, A. Glushchenko, D.-J. van Manen, and J. O. A. Robertsson, 2010, Interferometric ground-roll removal: Attenuation of scattered surface waves in single-sensor data: Geophysics, 75, no. 2, SA15-SA25

Haskell, N. A., 1953, The dispersion of surface waves on multilayered media: Bulletin of the Seismological Society of America, 43, 17-34.

He, R., B. Hornby, and G. Schuster, 2007, 3D wave-equation interferometric migration of VSP free-surface multiples: Geophysics, 72, no. 5, S195S203.

Henstridge, J. D., 1979, A signal processing method for circular arrays: Geophysics, 44, 179-184.

Hohl, D., and A. Mateeva, 2006, Passive seismic reflectivity imaging with ocean-bottom cable data: 76th Annual International Meeting, SEG, Expanded Abstracts, 1560-1563

Hornby, B. E., and J. Yu, 2007, Interferometric imaging of a salt flank using walkaway VSP data: The Leading Edge, 26, 760-763.

Jiang, Z., J. Sheng, J. Yu, G. T. Schuster, and B. E. Hornby, 2007, Migration methods for imaging different-order multiples: Geophysical Prospecting $\mathbf{5 5}, 1-19$.

Kang, T.-S., and J. S. Shin, 2006, Surface-wave tomography from ambient seismic noise of accelerograph networks in southern Korea: Geophysical Research Letters, 33, L17303-1-L17303-5.

Kennett, B. L. N., N. J. Kerry, and J. H. Woodhouse, 1978, Symmetries in the reflection and transmission of elastic waves: Geophysical Journal of the Royal Astronomical Society, 52, 215-230.

Kumar, M. R., and M. G. Bostock, 2006, Transmission to reflection transformation of teleseismic wavefields: Journal of Geophysical Research Solid Earth, 111, B08306-1-B08306-9.

Larose, E., A. Khan, Y. Nakamura, and M. Campillo, 2005, Lunar subsurface investigated from correlation of seismic noise: Geophysical Research Letters, 32, L16201-1-L16201-4.

Larose, E., L. Margerin, A. Derode, B. van Tiggelen, M. Campillo, N. Shapiro, A. Paul, L. Stehly, and M. Tanter, 2006, Correlation of random wave fields: An interdisciplinary review: Geophysics, 71, no. 4, SI11-SI21.

Le Bellac, M., F. Mortessagne, and G. G. Batrouni, 2004, Equilibrium and non-equilibrium statistical thermodynamics: Cambridge University Press.

Li, H., W. Su, C.-Y. Wang, and Z. Huang, 2009, Ambient noise Rayleigh wave tomography in western Sichuan and eastern Tibet: Earth and Planetary Science Letters, 282, 201-211.

Liang, C., and C. A. Langston, 2008, Ambient seismic noise tomography and structure of eastern North America: Journal of Geophysical Research Solid Earth, 113, B03309-1-B03309-18.

Lin, F.-C., M. P. Moschetti, and M. H. Ritzwoller, 2008, Surface wave tomography of the western United States from ambient seismic noise: Rayleigh and Love wave phase velocity maps: Geophysical Journal International, 173, 281-298.

Lin, F.-C., M. H. Ritzwoller, and R. Snieder, 2009, Eikonal tomography: Surface wave tomography by phase front tracking across a regional broadband seismic array: Geophysical Journal International, 177, 1091-1110.

Lobkis, O. I., and R. L. Weaver, 2001, On the emergence of the Green's function in the correlations of a diffuse field: Journal of the Acoustical Society of America, 110, 3011-3017.

Longuet-Higgins, M. S., 1950, A theory for the generation of microseisms: Philosophical Transactions of the Royal Society of London Series A, 243, $1-35$.

Lu, R., M. Willis, X. Campman, J. Ajo-Franklin, and M. N. Toksöz, 2008, Redatuming through a salt canopy and target-oriented salt-flank imaging: Geophysics 73, no. 3, S63-S71

Ma, S., G. A. Prieto, and G. C. Beroza, 2008, Testing community velocity models for southern California using the ambient seismic field: Bulletin of the Seismological Society of America, 98, 2694-2714.

Malcolm, A. E., J. A. Scales, and B. A. van Tiggelen, 2004, Extracting the Green function from diffuse, equipartitioned waves: Physical Review E, 70, 015601(R)-1-015601(R)-4.

Mercier, J.-P., M. G. Bostock, and A. M. Baig, 2006, Improved Green's functions for passive-source structural studies: Geophysics, 71, no. 4, SI95SI102.

Mikesell, D., K. van Wijk, A. Calvert, and M. Haney, 2009, The virtual refraction: Useful spurious energy in seismic interferometry: Geophysics, 74, no. 3, A13-A17.

Minato, S., K. Onishi, T. Matsuoka, Y. Okajima, J. Tsuchiyama, D. Nobuoka, H. Azuma, and T. Iwamoto, 2007, Cross-well seismic survey without borehole source: 77 th Annual International Meeting, SEG, Expanded Abstracts, 1357-1361.

Miyazawa, M., R. Snieder, and A. Venkataraman, 2008, Application of seismic interferometry to extract $\mathrm{P}$ - and $\mathrm{S}$-wave propagation and observation of shear-wave splitting from noise data at Cold Lake, Alberta, Canada: Geophysics, 73, no. 4, D35-D40.
Nowack, R. L., S. Dasgupta, G. T. Schuster, and J.-M. Sheng, 2006, Correlation migration using Gaussian beams of scattered teleseismic body waves: Bulletin of the Seismological Society of America, 96, 1-10.

Okada, H., 2003, The microtremor survey method: SEG.

, 2006, Theory of efficient array observations of microtremors with special reference to the SPAC method: Exploration Geophysics, 37, 73-85.

Perton, M., F. J. Sánchez-Sesma, A. Rodríguez-Castellanos, M. Campillo, and R. L. Weaver, 2009, Two perspectives on equipartition in diffuse elastic fields in three dimensions: Journal of the Acoustical Society of America, 126, 1125-1130.

Picozzi, M., S. Parolai, D. Bindi, and A. Strollo, 2009, Characterization of shallow geology by high-frequency seismic noise tomography: Geophysical Journal International, 176, 164-174.

Poletto, F., and L. Petronio, 2003, Transmitted and reflected waves in tunnel SWD: 73rd Annual International Meeting, SEG, Expanded Abstracts, $1211-1214$

,2006, Seismic interferometry with a TBM source of transmitted and reflected waves: Geophysics, 71, no. 4, SI85-SI93.

Ramírez, A. C., and A. B. Weglein, 2009, Green's theorem as a comprehensive framework for data reconstruction, regularization, wavefield separation, seismic interferometry, and wavelet estimation: A tutorial: Geophysics, 74, no. 6, W35-W62.

Rickett, J., and J. Claerbout, 1999, Acoustic daylight imaging via spectral factorization: Helioseismology and reservoir monitoring: The Leading Edge, 18, 957-960.

Rondenay, S., M. G. Bostock, and J. Shragge, 2001, Multiparameter two-dimensional inversion of scattered teleseismic body waves. 3 . Application to the Cascadia 1993 data set: Journal of Geophysical Research, 106, 3079530807.

Roux, P., and M. Fink, 2003, Green's function estimation using secondary sources in a shallow water environment: Journal of the Acoustical Society of America, 113, 1406-1416.

Rytov, S. M., 1956, On thermal fluctuations in distributed systems: Doklady Akademii Nauk SSSR, 110, 371

Rytov, S. M., Y. A. Kravtsov, and V. I. Tatarskii, 1989, Principles of statistical radiophysics. 3: Elements of random fields: Springer-Verlag Berlin

Sabra, K. G., P. Gerstoft, P. Roux, W. A. Kuperman, and M. C. Fehler, 2005a, Extracting time-domain Green's function estimates from ambient seismic noise: Geophysical Research Letters, 32, L03310-1-L03310-5.

, 2005b, Surface wave tomography from microseisms in southern California: Geophysical Research Letters, 32, L14311-1-L14311-4.

Sabra, K. G., P. Roux, and W. A. Kuperman, 2005c, Arrival-time structure of the time-averaged ambient noise cross-correlation function in an oceanic waveguide: Journal of the Acoustical Society of America, 117, no. 1, 164174.

Sánchez-Sesma, F. J., J. A. Pérez-Ruiz, M. Campillo, and F. Luzón, 2006, Elastodynamic 2D Green function retrieval from cross-correlation: $\mathrm{Ca}-$ nonical inclusion problem: Geophysical Research Letters, 33, L13305-1L13305-6.

Scherbaum, F., 1987a, Seismic imaging of the site response using microearthquake recordings. Part I. Method: Bulletin of the Seismological Society of America, 77, 1905-1923.

_ 1987b, Seismic imaging of the site response using microearthquake recordings. Part II. Application to the Swabian Jura, southwest Germany, seismic network: Bulletin of the Seismological Society of America, 77, 1924-1944.

Schuster, G. T., 2001, Theory of daylight/interferometric imaging: Tutorial: 63rd Conference \& Technical Exhibition, EAGE, Extended Abstracts, A32.

- 2009, Seismic interferometry: Cambridge University Press.

Schuster, G. T., J. Yu, J. Sheng, and J. Rickett, 2004, Interferometric/daylight seismic imaging: Geophysical Journal International, 157, 838-852.

Schuster, G. T., and M. Zhou, 2006, A theoretical overview of model-based and correlation-based redatuming methods: Geophysics, 71, no. 4, SI103SI110.

Shapiro, N. M., and M. Campillo, 2004, Emergence of broadband Rayleigh waves from correlations of the ambient seismic noise: Geophysical Research Letters, 31, L07614-1-L07614-4.

Shapiro, N. M., M. Campillo, L. Stehly, and M. H. Ritzwoller, 2005, Highresolution surface-wave tomography from ambient seismic noise: Science, 307, 1615-1618.

Shragge, J., B. Artman, and C. Wilson, 2006, Teleseismic shot-profile migration: Geophysics, 71, no. 4, SI221-SI229.

Shragge, J., M. G. Bostock, and S. Rondenay, 2001, Multiparameter two-dimensional inversion of scattered teleseismic body waves. 2. Numerical examples: Journal of Geophysical Research, 106, 30783-30793.

Snieder, R., 2004, Extracting the Green's function from the correlation of coda waves: A derivation based on stationary phase: Physical Review E, 69, 046610-1-046610-8.

Snieder, R., K. Wapenaar, and U. Wegler, 2007, Unified Green's function retrieval by cross-correlation: Connection with energy principles: Physical 
Review E, 75, 036103-1-036103-14.

Stehly, L., M. Campillo, and N. M. Shapiro, 2006, A study of the seismic noise from its long-range correlation properties: Journal of Geophysical Research, 111, B10306-1-B10306-12.

Thompson, A. R., J. M. Moran, and G. W. Swenson Jr., 2001, Interferometry and synthesis in radio astronomy: Wiley Interscience.

Thomson, W. T., 1950, Transmission of elastic waves through a stratified solid medium: Journal of Applied Physics, 21, 89-93.

Toksöz, M. N., 1964, Microseisms and an attempted application to exploration: Geophysics, 39, 154-177.

Tonegawa, T., K. Nishida, T. Watanabe, and K. Shiomi, 2009, Seismic interferometry of teleseismic S-wave coda for retrieval of body waves: An application to the Philippine Sea slab underneath the Japanese Islands: Geophysical Journal International, 178, 1574-1586.

Torii, K., T. Matsuoka, K. Onishi, K. Shirashi, T. Aizawa, Y. Yamanaka, S. Ito, T. Kimura, Y. Asano, and T. Takeda, 2007, Application of seismic interferometry to natural earthquakes measured by small-scale array: 77th Annual International Meeting, SEG, Expanded Abstracts, 1362-1366.

Ursin, B., 1983, Review of elastic and electromagnetic wave propagation in horizontally layered media: Geophysics, 48, 1063-1081.

van Tiggelen, B. A., 2003, Green function retrieval and time reversal in a disordered world: Physical Review Letters, 91, 243904-1-243904-4.

Wang, Y., Y. Luo, and G. T. Schuster, 2009, Interferometric interpolation of missing seismic data: Geophysics, 74, no. 3, SI37-SI45.

Wapenaar, K., D. Draganov, J. Thorbecke, and J. Fokkema, 2002, Theory of acoustic daylight imaging revisited: 72nd Annual International Meeting, SEG, Expanded Abstracts, 2269-2272.

Wapenaar, K., and J. Fokkema, 2006, Green's function representations for seismic interferometry: Geophysics, 71, no. 4, SI33-SI46.

Wapenaar, C. P. A., and J. L. T. Grimbergen, 1996, Reciprocity theorems for one-way wave fields: Geophysical Journal International, 127, 169-177.

Wapenaar, K., J. Thorbecke, and D. Draganov, 2004, Relations between reflection and transmission responses of three-dimensional inhomogeneous media: Geophysical Journal International, 156, 179-194.

Weaver, R., B. Froment, and M. Campillo, 2009, On the correlation of nonisotropically distributed ballistic scalar diffuse waves: Journal of the
Acoustical Society of America, 126, 1817-1826.

Weaver, R. L., and O. I. Lobkis, 2001, Ultrasonics without a source: Thermal fluctuation correlations at MHz frequencies: Physical Review Letters, 87, 134301-1-134301-4.

,2002, On the emergence of the Green's function in the correlations of a diffuse field: Pulse-echo using thermal phonons: Ultrasonics, 40, 435-439.

Webb, S. C., 1998, Broadband seismology and noise under the ocean: Reviews of Geophysics, 36, 105-142.

Willis, M. E., R. Lu, X. Campman, M. N. Toksöz, Y. Zhang, and M. V. de Hoop, 2006, A novel application of time-reversed acoustics: Salt-dome flank imaging using walkaway VSP surveys: Geophysics, 71, no. 2, A7A11.

Xiao, X., M. Zhou, and G. T. Schuster, 2006, Salt-flank delineation by interferometric imaging of transmitted P- and S-waves: Geophysics, 71, no. 4, SI197-SI207.

Xue, Y., S. Dong, and G. T. Schuster, 2009, Interferometric prediction and subtraction of surface waves with a nonlinear local filter: Geophysics, $\mathbf{7 4}$ no. 1, SI1-SI8.

Yao, H., X. Campman, M. V. de Hoop, and R. D. van der Hilst, 2009, Estimation of surface wave Green's functions from correlation of direct waves, coda waves, and ambient noise in SE Tibet: Physics of the Earth and Planetary Interiors, 177, 1-11.

Yao, H., R. D. van der Hilst, and M. V. de Hoop, 2006, Surface-wave array tomography in SE Tibet from ambient seismic noise and two-station analysis - I. Phase velocity maps: Geophysical Journal International, 166, 732-744.

Yao, H., C. Beghein, and R. D. van der Hilst, 2008, Surface-wave array tomography in SE Tibet from ambient seismic noise and two-station analysis - II. Crustal and upper-mantle structure: Geophysical Journal International, 173, 205-219.

Yokoi, T., and S. Margaryan, 2008, Consistency of the spatial autocorrelation method with seismic interferometry and its consequence: Geophysical Prospecting, 56, 435-451.

Yu, J., and G. T. Schuster, 2006, Crosscorrelogram migration of inverse vertical seismic profile data: Geophysics, 71, no. 1, S1-S11. 